\title{
Solve traveling salesman problem using EMF-CE algorithm
}

This paper was downloaded from TechRxiv (https://www.techrxiv.org).

\section{LICENSE}

CC BY 4.0

SUBMISSION DATE / POSTED DATE

$24-10-2020$ / 28-10-2020

CITATION

Luo, Meng; Gu, Shiliang (2020): Solve traveling salesman problem using EMF-CE algorithm. TechRxiv. Preprint. https://doi.org/10.36227/techrxiv.13139042.v2

$\mathrm{DOI}$

10.36227/techrxiv.13139042.v2 


\section{Solve traveling salesman problem using EMF-CE algorithm}

Meng Luo ${ }^{\mathrm{a} *}$, Shiliang $\mathrm{Gu}^{\mathrm{a} *}$

a Jiangsu Provincial Key Laboratory of Crop Genetics and Physiology/Co-Innovation Center for Modern Production Technology of Grain Crops; Key Laboratory of Plant Functional Genomics of Ministry of Education; Wheat Research Center, Yangzhou University, Yangzhou, Jiangsu 225009, China

* Corresponding author.

E-mail addresses: slgu@yzu.edu.cn (Shiliang Gu); czheluo@gmail.com (Meng Luo);

Abstract-In this paper, a novel search algorithm that based on the Contraction-Expansion algorithm and integrated three operators Exchange, Move, and Flip (EMF-CE) is proposed for the traveling salesman problem (TSP). EMF-CE uses a negative exponent function to generate critical value as the feedback regulation of algorithm implementation. Also, combined Exchange Step, Move step with Flip step and constitute of more than twenty combinatorial optimizations of program elements. It has been shown that the integration of local search operators can significantly improve the performance of EMF-CE for TSPs. We test small and medium scale (51-1000 cities) TSPs were taken from the TSPLIB online library. The experimental results show the efficiency of the proposed EMF-CE for addressing TSPs compared to other state-of-the-art algorithms.

Index Terms - Contraction-Expansion algorithm (CE), Exchange-Move-Flip (EMF), combinatorial optimization, traveling salesman problem (TSP)

\section{I .INTRODUCTION}

The traveling salesman problem (TSP) is well known for the classical and fundamental NP-hard combinatorial optimization problems. The classical TSP that can be described as following: find a path through a weighted graph that starts and ends in the same city, includes every other city exactly once and minimizes the total distance tour of $\mathrm{n}$ cities. The above-described path is always a Hamiltonian cycle or tour. In the mathematical field of graph theory, the Hamiltonian path problem and the Hamiltonian cycle problem are problems of determining whether a Hamiltonian path or a Hamiltonian cycle exists in a given graph (whether directed or undirected). Both problems are NP-complete [1-3]. Special cases of the TSP that included metric TSP, Euclidean TSP, asymmetric TSP, analyst's traveling salesman problem, and TSP path length for random sets of points in a square. TSPs raise important issues because various problems in 
science, engineering, and bioinformatics fields such as very large scale integration(VLSI) design[4], vehicle routing[5], scheduling problems[6], integrated circuits designs[7], genome sequencing[8, 9], physical mapping problems[10], and constructing phylogenetic trees[11] can be formulated as TSPs. An important characteristic of these problems is that tests performed on challenging TSP instances provide a basis for analyzing the performance characteristics of global search meta-heuristic techniques[2]. On the other hand, it falls into a class of NP-hard or NP-complete problems. Therefore, the research on the TSP is theoretically important. This paper focuses on a Contraction-expansion algorithm based on Move, Change, and Fliplr technique that generates near to optimal TSP solutions.

After all these years, a huge number of methods have been developed for solving TSPs. Exact methods[12], heuristics[3, 13-15], and metaheuristics[16-19] have been proposed to solve the static TSPs. Due to the fierce increase in the computation time when the problem size increases, heuristics and metaheuristics are more preferable than exact methods since they trade optimality for efficiency. And also the TSP has attracted extensive research and has been repeatedly used as the basis of comparison for different optimization algorithms such as genetic algorithms (GAs)[19, 20], tabu search[21-24], simulated annealing(SA)[25, 26], local search[27, 28], ant colony system(ACS)[16, 29], evolutionary algorithms (EAs)[18], neural network[30,31], and so on. These diverse approaches have demonstrated various degrees of strength and success.

Most exact methods, e.g., Concorde, and heuristic methods, e.g., Lin-Kernighan, 2-Opt or 3-Opt, are not (directly) applicable for asymmetric TSP cases since they are based on the triangle inequality of Euclidean distance associated with symmetric TSPs. Methods for asymmetric TSPs are less studied with only a few exceptions $[32,33]$. Of course, symmetric methods can be applied with some modifications to the problem or the method itself. But, this may significantly increase the computation time or degrade their effectiveness [29], [52]. Evolutionary algorithms(EAs) and Ant colony optimization (ACO) algorithms have proved to be powerful methods to tackle such problems due to their adaptation capabilities. Such methods are capable of finding the global optimum (or close to the global optimum) solution for symmetric TSP cases in seconds.

Recently, there were many new approaches derived from evolutionary algorithms(EAs), ant colony optimization (ACO), simulated annealing(SA), and neural network, which have been presented effectually to solve the TSPs[18, 25, 26, 34-36]. Such as Genetic-based Particle Swarm Optimization (GPSO) algorithm for TSPs, and this evolutionary algorithm includes two phases. Where the first two was fuzzy CMeans and Genetic-based PSO procedure applied to reduce the complexity and better efficiency of solving TSPs with large number of cities, respectively[37]. Another two methods that hybrid method (based on Particle Swarm Optimization, Ant Colony Optimization, and 3-Opt algorithms) and adaptive simulated 
annealing algorithm with greedy search to solve TSP, which was better than the former of the performance $[25,38]$. In hybrid methods that PSO is used for determining parameters, and which affected the performance of the ACO, and the 3-Opt is used for getting rid of the local solution found in the ACO algorithm. The metaheuristics approach looks for more effective mutations, a more rational greedy search strategy, and more universal adaptive parameter control of the proposed algorithm to achieve better results and faster convergence in solving TSP with large-scale cities. The solution accuracy of the Move, Change and Flip Search Algorithm(MCFSA) are still not comparable to some other state-of-the-art heuristics and metaheuristics for the TSP, including Ant Colony Optimization, Simulated Annealing[25], and Evolutionary algorithms(EAs) [18] for tackling the large-scale cities.

The rest of this paper is organized as follows. Section II introduces the TSP problem definition and describes how the TSP cases are generated. Section III describes the approaches from the literature that have been applied to solve the TSPs. Section IV gives details of the proposed EMF-CE algorithm. Section $\mathrm{V}$ presents the experimental studies that include comparisons with other popular heuristics and state-of-theart algorithms. Finally, concluding remarks and future work directions are presented in Section VI.

\section{II .DEFINITION OF TRAVELING SALESMAN PROBLEMS}

\section{A.Problem Formulation}

Quintessentially, a TSP instance is modeled by a fully connected weighted graph $G=(\mathrm{N}, \mathrm{A})$, where $N=\left\{x_{1}, \gg, x_{n}\right\}$ is a set of $\mathrm{n}$ nodes and $A=\left\{\left(x_{i}, x_{j}\right)\left|x_{i}, x_{j}\right| \mathbf{f}, i j\right\}$ is a set of arcs. For the classic TSP, nodes and arcs represent the cities and the links between them. Each arc $\left(x_{i}, x_{j}\right)$ Í $A$ is associated with a nonnegative value $d_{i j}$ I $R^{+}$, which for the classic TSP represents the distance between cities $x_{i}$ and $x_{j}$. While TSP instance is considered to be symmetric if $d_{i j}=d_{i j}$, " $\left(x_{i}, x_{j}\right)$ í , or asymmetric if $d_{i j}, d_{i j}$ for at least one $\left(x_{i}, x_{j}\right)$ Í $A$, and in this paper we only considered the symmetric TSP. The most studied and well known special case of symmetric TSP is the version in which the distances among cities always satisfy the triangle inequality (e.g., $d_{i j}+d_{j k}{ }^{2} d_{i k}$ ), known as Euclidean TSP. But the definition of $d_{i j}$ that the distance among cities may have multiple choices, while we also used the Euclidean distance here. Currently, there are many existing methods, both exact and heuristic, such that are based on the triangle inequality [12]. However, the corresponding asymmetric special case may not always satisfy the triangle inequality. 
Evaluation function: It measures the cost of the route and is calculated using the sum of the Euclidean distances between each pair of cities with coordinates $\left(x_{i}, x_{j}\right)$. Assumed that the route has a length of $\mathrm{n}$ cities, then the fitness function[39] is defined in this paper as follows:

$$
\operatorname{tsp}(x)=\ddot{a}_{i=1}^{n-1} d\left(x_{i}, x_{j}\right) \quad H\left(x_{1}, x_{n}\right), j \quad i=1
$$

Where $d\left(x_{i}, x_{j}\right)$ is the distance between cities $x_{i}$ and $x_{j}$. If both cities have $y$ and $z$ coordinates, then the distance is calculated as:

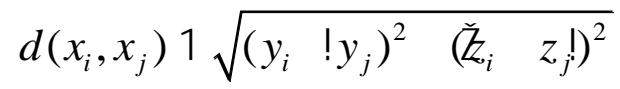

\section{III.METHODOLOGIES FOR SOLVING TSPs}

The challenges of algorithms in solving NP-complete problems and in static environments are wellknown in terms of computational complexity. In the following sections, we briefly review existing and comparing algorithms designed to address the TSPs.

\section{A. Evolutionary Algorithms}

Evolutionary algorithms (EAs) forebode a very promising direction. However, only general problemindependent EAs are frequently inefficient in solving TSPs, especially large TSPs. Tsai et al.[18] proposes an evolutionary algorithm, called the heterogeneous selection evolutionary algorithm (HeSEA), which integrates edge assembly crossover (EAX) and Lin-Kernighan (LK) local search, through family competition and heterogeneous pairing selection. Where the HeSEA is especially efficient to cope with the large TSPs.

Jin et al.[19] proposed the integrated self-organizing map (ISOM) that combination the canonical genetic algorithm(GA) in the neural-evolutionary system, in which every individual (chromosome) represents an effective learning scheme(rule). The evolved ISOM (eISOM) has made at least a $1 \%$ improvement over the SOM developed by Budinich[30], the ESOM[40], the convex elastic net[41], and the FLEXMAP[42] algorithm that examined on a wide spectrum of TSPs. Feng et al. [43] developed the hybrid evolutionary fuzzy learning scheme combines the adaptive fuzzy C-means (FCM), simple MAX-MIN merging concept, simulated annealing (SA) learning algorithm, and an efficient table transform-based particle swarm optimization (TPSO) that automatically determines the near-optimal traveling path in LSTSPs. It denotes the clustering-based FCM algorithm as a classifier to reduce the complexity of the full traveling cities by dividing samples into the proper number of subgroups. After the TPSO and SA local optimal learning scheme identify the optimal traveling paths in each subgroup, and the novel and effective MAX-MIN algorithm rebuilds the complete traveling path. 
Baraglia et al.[44] showed that combines the compact genetic algorithm(Cga) for the TSP with an efficient implementation of LK local search heuristics designed by Lin and Kernighan[14](Cga-LK). Which exploited the low memory requirements and the high computational efficiency. Nguyen et al.[45] further proposed hybrid genetic algorithm (HGA) was based on a multipopulation steady-state GA and combined with the local search heuristics. The offspring replacing the worst individual in the population that using a steady-state update strategy and linear ranking for selecting parents to maintain a more diverse population for a GA. They also use LK local search heuristics for tour improvement. And these approaches aimed at solving the large-scale TSPs.

\section{B. Simulated Annealing}

Simulated annealing (SA) is a probabilistic technique for approximating the global optimum of a given function. Specifically, it is a metaheuristic to approximate global optimization in a large search space. It is often used when the search space is discrete (e.g., all tours that visit a given set of cities)[7]. But the major shortage of SA is that it can be extremely slow and require significantly more processing time than other meta-heuristics. Several studies have tried to improve SAs performance by changing the generation and acceptance mechanisms.

Pepper et al.[46] applied a conventional simulated annealing(SA) algorithm to solve Euclidean TSPs, which SA showed as the best of the non-demon algorithms with average accuracy and running time. Geng et al.[25] proposed an adaptive simulated annealing algorithm with greedy search (ASA-GS) to deal with the TSPs, and based on the standard simulated annealing that it can find a better solution at the cost of vast time. Chen et al.[26] presented a method that called the genetic simulated annealing ant colony system with particle swarm optimization techniques for solving the TSPs. The system simulates the annealing process of metal atoms. Metal atoms at a high temperature will become unstable from their initial states and search for the newest states. Li et al.[47] proposed a new hybrid scheme of parallel tempering and simulated annealing (hybrid PT/SA) to copy with the TSPs. Which the SA process was to allow the temperature at each temperature level to decrease gradually toward a target temperature.

Wang et al.[48] proposed a multi-agent SA algorithm with instance-based sampling (MSA-IBS) aims at both improving the efficiency of SA and adding parallelism of SA more naturally for traveling salesman problem, which employs a population of agents running SA algorithm and evolving individuals of instancebased meta-heuristics collaboratively. Motivated by the success of using instance-based learning to improve the efficiency of SA algorithm $[49,50]$ and ACO algorithm. Wang et al[51] presented swarm SA 
(SSA) algorithm which exploited swarm of agents running SA algorithm and used the solution component selection scheme of ACO algorithm to generate candidate solutions that can be efficient solve the TSPs.

Inspired by a metaheuristic algorithm called the list-based threshold-accepting (LBTA) algorithm which has shown significant performance for combinatorial optimization problems that are NP-hard[52, 53]. Zhan et al.[54] proposed the list-based simulated annealing (LBSA) algorithm to solve traveling salesman problem algorithm, and used the novel list-based cooling scheme to control the decrease of temperature, also generated a list of maximum temperature which was used by Metropolis acceptance criterion to decide whether to accept the candidate solution.

\section{Ant Colony Optimization Algorithms}

Ant colony optimization algorithm (ACO) is a probabilistic technique for solving computational problems which can be reduced to discovery good paths through graphs. However, for multifaceted largescale optimization problems, the drawback of the ACO that was leisurely premature convergence.

The AS[55] is the first ACO algorithm applied for the TSP. Dorigo et al.[16] proposed ant colony system (ACS) algorithm that built on the previous ant system in the direction of enlightening efficiency when applied to symmetric and asymmetric TSPs. The main idea that was sets of cooperating agents called ants, which search in parallel for good solutions to the TSP and also cooperated through pheromone-mediated indirect and global communication. They also combined the local optimization heuristic with the restricted

3-opt procedure, which inserting/removing three edges on the path, and considers only 3-opt moves that did not return the order in which the cities were visited. Furthermore, also Chu et al.[56] presented the parallel ant colony system that combined seven communication strategies, which the communication was to update the pheromone level for each route according to the best route found by neighboring groups. However, Chen et al.[57] further built on the PACS and cooperating with GA, while the PGACS outperformed the PACS in terms of accuracy solution quality.

Mavrovouniotis and Yang [58] further improved the MMAS algorithm[59] based on with or without direct communication(DC) scheme, where the DC scheme reduced computational runtime and improved the solution quality of conventional ACO algorithms to solve the large-scale TSPs. Which DC scheme allowing to exchange cities and utilize the different solutions constructed by ants on each iteration.

\section{Heuristics And Neural Network}

Local search with k-change neighborhoods, k-opt, are the most widely used heuristic method for the traveling salesman problem. Helsgaun $[60,61]$ proposed a new LKH called LKH-2, and based on the LKH1. which eliminates many of the limitations and shortcomings LHK-1. Combined use of general K-opt 
moves with six partitioning schemes, for obtaining high-quality solutions and reducing the complexity of solving large-scale problem instances, respectively. As we all know, LKH-2 is the best search algorithm to achieve the best optimal tour so far. Rego et al.[15] surveyed leading heuristics for the TSPs, which gathered with Lin-Kernighan (LK) and stem-and-cycle (S\&C) methods, as well known the most effective and efficient local search ejection chain (EC) methods. Proposing six and two variants of the LinKernighan (LK) and stem-and-cycle (S\&C) ejection chain method, respectively.

Mahdi Saadatmand-Tarzjan et al.[62] proposed a novel constructive-optimizer NN (CONN), combined uses a feedback structure and competitive training algorithm, which similar to Hopfield-type NNs $(\mathrm{HNN})[63]$ and Kohonen-type self-organizing maps(K-SOM)[19] were presented to provide the best conciliation between the convergence speed and solution quality, respectively. Consequently, CONN has consisted of a constructive part, which grew the tour and an optimizer part to optimize it. In the training algorithm, an initial tour was created first and introduced to CONN. Thiago A.S. Masutti et al.[36] further modified the Real-Valued Antibody Network designed to solve the Traveling Salesman Problem (RABNET-TSP) [64], which cooperated with the immune-inspired self-organizing neural network based on the immune system, and aim at improving its efficacy (quality of the solutions found) and reducing the computational time.

\section{PROPOSED EXCHANGE-MOVE-FLIP BASED ON CE ALGORITHM}

In this paper, a novel search algorithm that based on we proposed Contraction-Expansion algorithm[65] is introduced to provide the best compromise between the convergence speed and solution quality. The main idea of the proposed EMF-CE is taking advantage of integrated three operators Move, Exchange, and Flip techniques, and cooperated the CE to search for the optimal solution of TSPs. We will use ten nodes as an example to illustrate the process of the EMF-CE algorithm, as Fig.1-a show. 

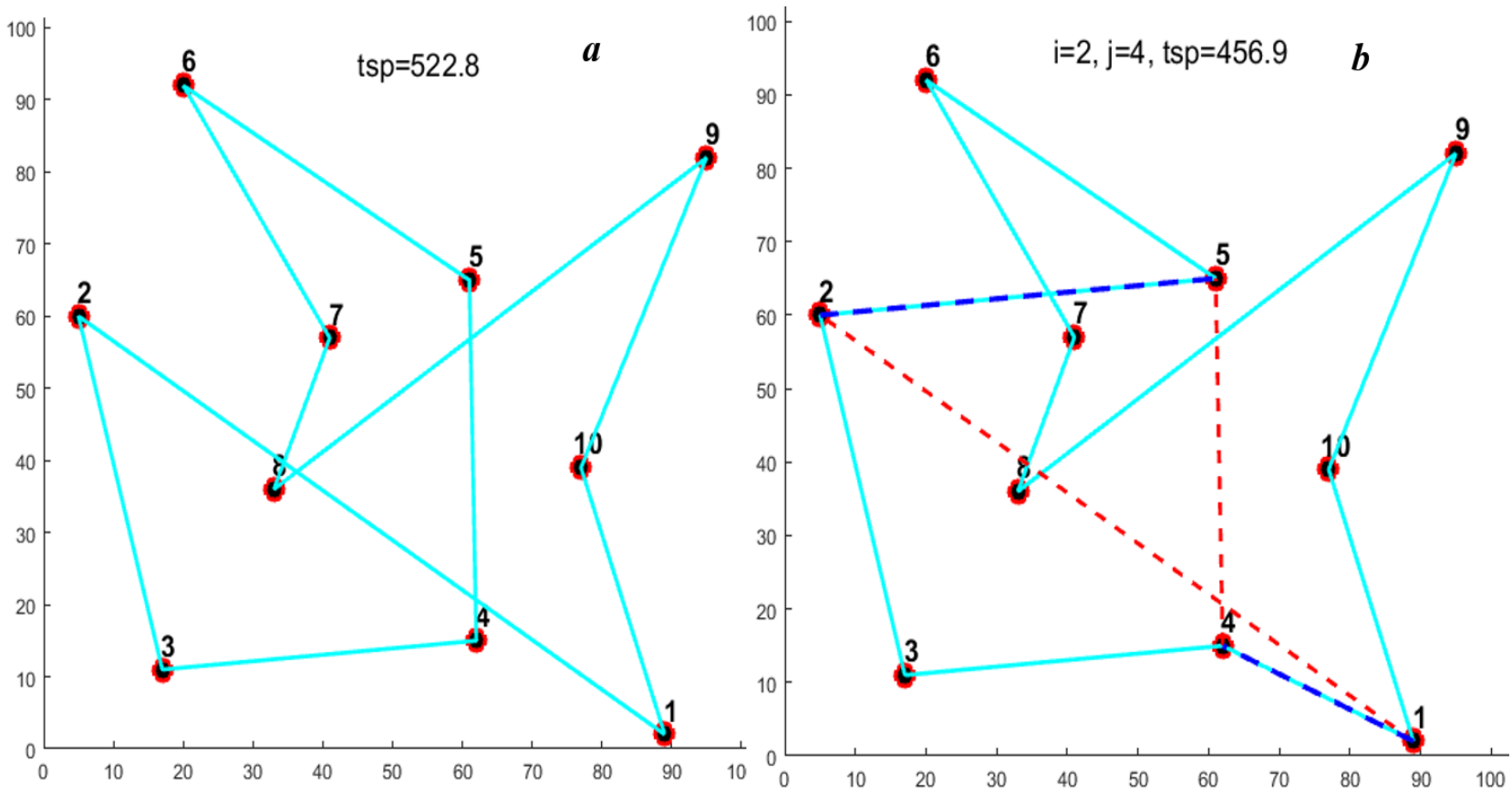

Fig 1 the original path of ten nodes (a) and exchange $x_{2}$ and $x_{4}(\mathrm{~b})$

\section{A.Exchange Step}

Assume that have $x_{1}, \gg, x_{n}$ nodes, if exchange $x_{i}$ and $x_{j}(i \neq j=1 \ldots n)$ can make the total distance $t s p(1)$ value less than before, then achieved this exchange step, furthermore, anew generated another better tour. Those exchanges should include that the position of $x_{i}$ and $x_{j}$ in a two-dimensional array-based data structure with an n-vector to control two-dimensional matrix $(n \times 2)$ original data. Each row corresponds to each current city and each column corresponds to the order of traveling sequence of this current tour. Also, the position wherein the Euclidean distance matrix the row and column of $x_{i}$ and $x_{j}$ will be complete exchange. To illustrate how this Exchange step is formulated, the following example is used for explanation. Which there are ten cities(nodes), as Fig.1b, after exchanged the $x_{2}$ and $x_{4}$, the original path sequence 1-2-3-4-5... became 1-4-3-2-5... The difference of tour length between the old path and the new path as follows:

$$
d=d(1,2) \quad H(4,5) \quad d(1,4) \quad d(2,5) \quad 659
$$

Where $d<0$, it is indicated that the new path superior old path, then exchanged $x_{2}$ and $x_{4}$ two nodes. Here, if $d \nsubseteq 2$, then $d$ that the difference of tour length only is between the red and blue dashed lines Fig1b. And the other line segments (arcs) without change. Identically, it isn't necessary to calculate the distance in order to that decrease the computation burden. If $d>2$, which would be involved eight line segments (arcs) (Fig.2a-b and Fig3a-b, the four red and four blue dashed lines). The difference in tour length $d$ should be the following: 


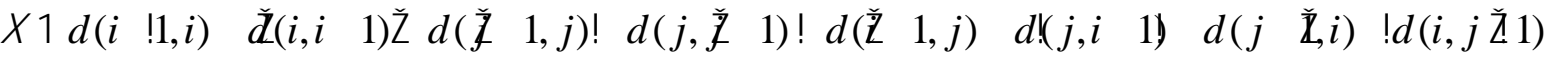

$$
i=2,3, \quad \gg, n-1, j \quad i=1, i+2,+, n \gg
$$
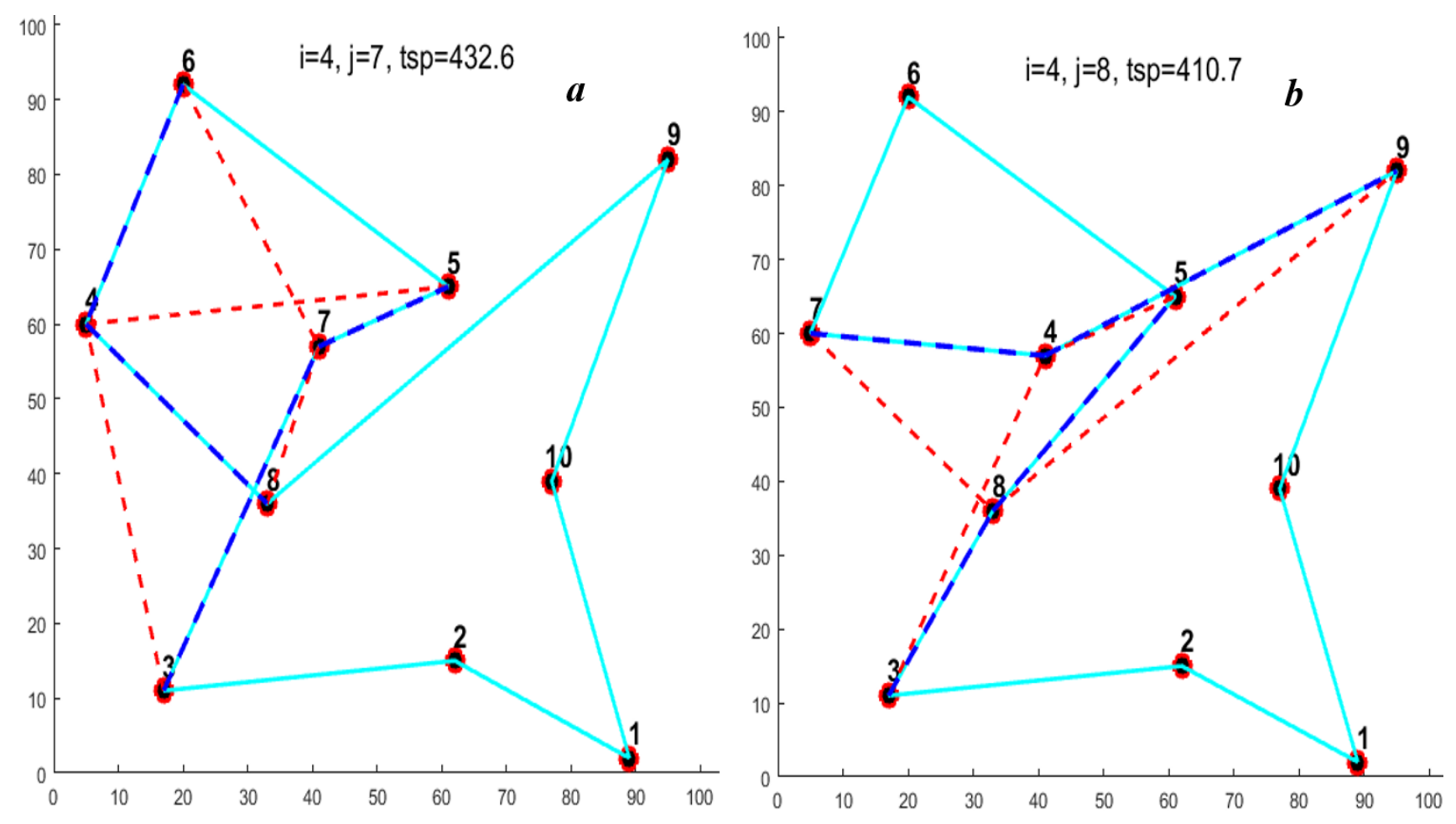

Fig. 2 exchange $x_{4}, x_{7}$ and $x_{4}, x_{8}$ nodes

It is worth noting that should be immediately exchanged only if $d<0$. Meanwhile, exchange the row 242 position of $x_{i}$ and $x_{j}$ in two-dimensional matrix of original data, also corresponding to exchange row and 243 column in the distance matrix. The exchanged sequence can from the smallest to largest (such an exchange 244 was called sequential[66], $i$ was small to large, $j>i$ ) executed or inverse ( $i$ was large to small, $j<i)$ executed. 245 Identically, for the next Move step and Flip step both should be executed and following this principle. 246 Given a feasible TSP tour, the algorithm repeatedly performs exchanges that reduce the length of the current tour, until a tour is reached for which no exchange yields an improvement. 

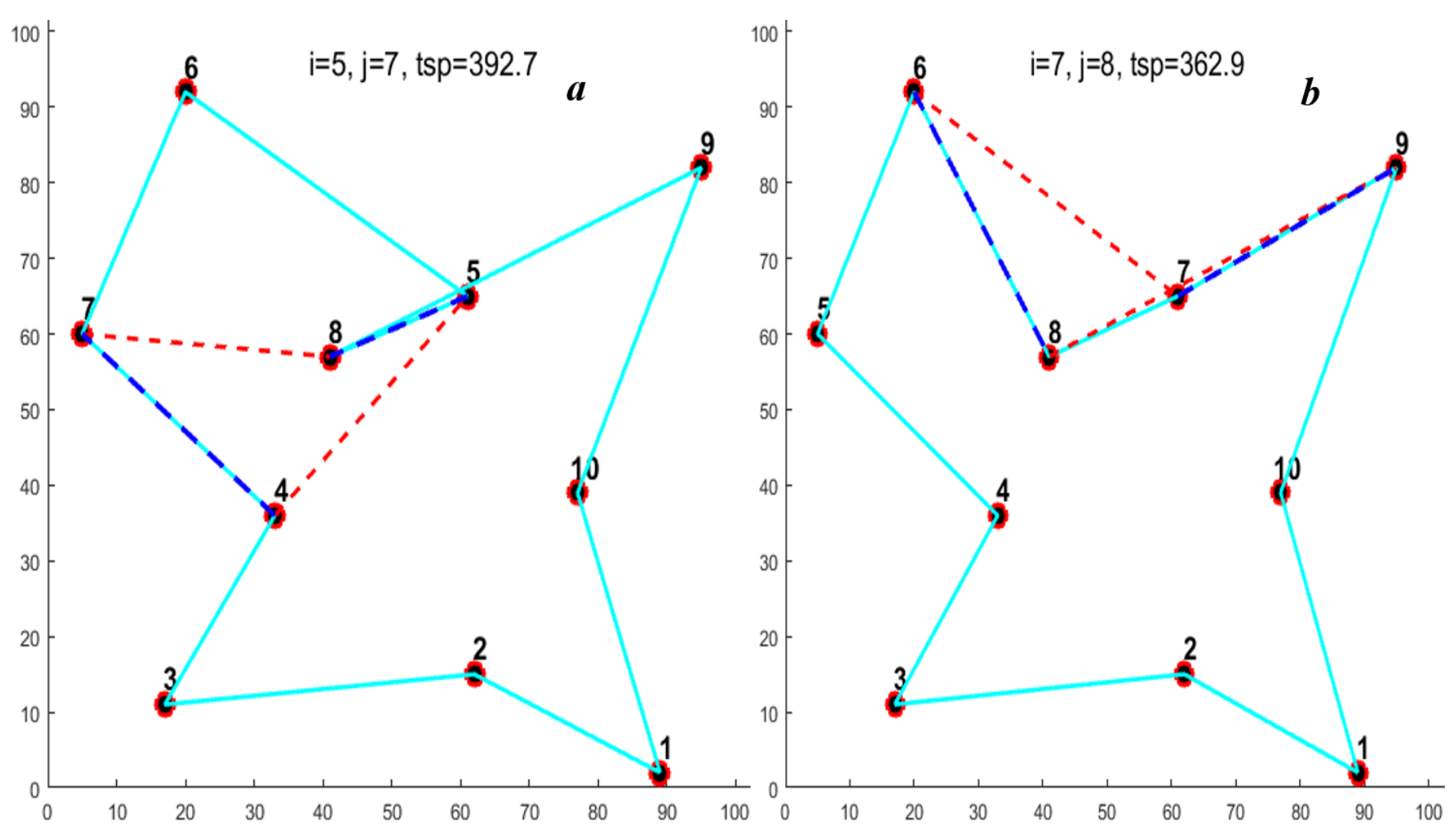

Fig.3 exchange $x_{5}, x_{7}(\mathrm{a})$ and $x_{7}, x_{8}(\mathrm{~b})$ nodes

All possible tours are made of ten nodes that more than one hundred and eighty thousand. But in the original tour of ten nodes (the sequence was random permutation), where only exchanged 5 times and instantly achieved optimal result Fig.1a-b. In the completely random (or random permutation nodes) condition, where the exchange times will be an increase, while will not considerably increase, and also can achieve preferably (maybe optimal) result. If found the better tour or smallest tsp (1) value should be marker Xm and $t$ spm variables archived. Algorithm1-7 (supplement1).

\section{B.Move Step}

k-Opt algorithm (2-, 3-, and 4-opt move, etc.) was a simple local search algorithm for solving the TSP in optimization[66]. In this algorithm, k-Opt analysis involves deleting k connections (or edges) on a tour, reconnecting the tour in all other possible ways, and then evaluating each reconnection method to find the optimum one. Here, we proposed the move (1-move, 2-move, 3-move, etc.) algorithm of concept was different from k-opt, which only moves the nodes forward or backward, and also can move one, two, and three nodes etc together. For instance, tried to move the position of $x_{i}(i=1 \ldots n)$ forward or backward, and only change it that in the sequence of the TSP (Traveling salesman problems) tour. If made the $t s p(1)$ value less than before, then executed this step. The number of move nodes could be one node or simultaneously moved to adjoin nodes from two to n nodes were chose. Buts large number of experiments show that move simultaneously chose from two to five nodes together was the best choice. Such as, based on the original of 
ten nodes path Fig.4a, and moved $x_{3}$ to the front of $x_{2}$ Fig.4b (this step is equivalent to exchange $x_{3}$ and $x_{2}$, both moves adjoin nodes are equivalent to exchange step), and the difference of tour length that the old path and generated new path only included four segments Fig.4b (two red dashed line and two blue dashed line) (5). Cause $d<0$, this move step made the TSP path shorter.

$$
d=d(1,3) \quad H(2,4) \quad d(1,2) \quad d(3,4) \quad 2 \neq
$$
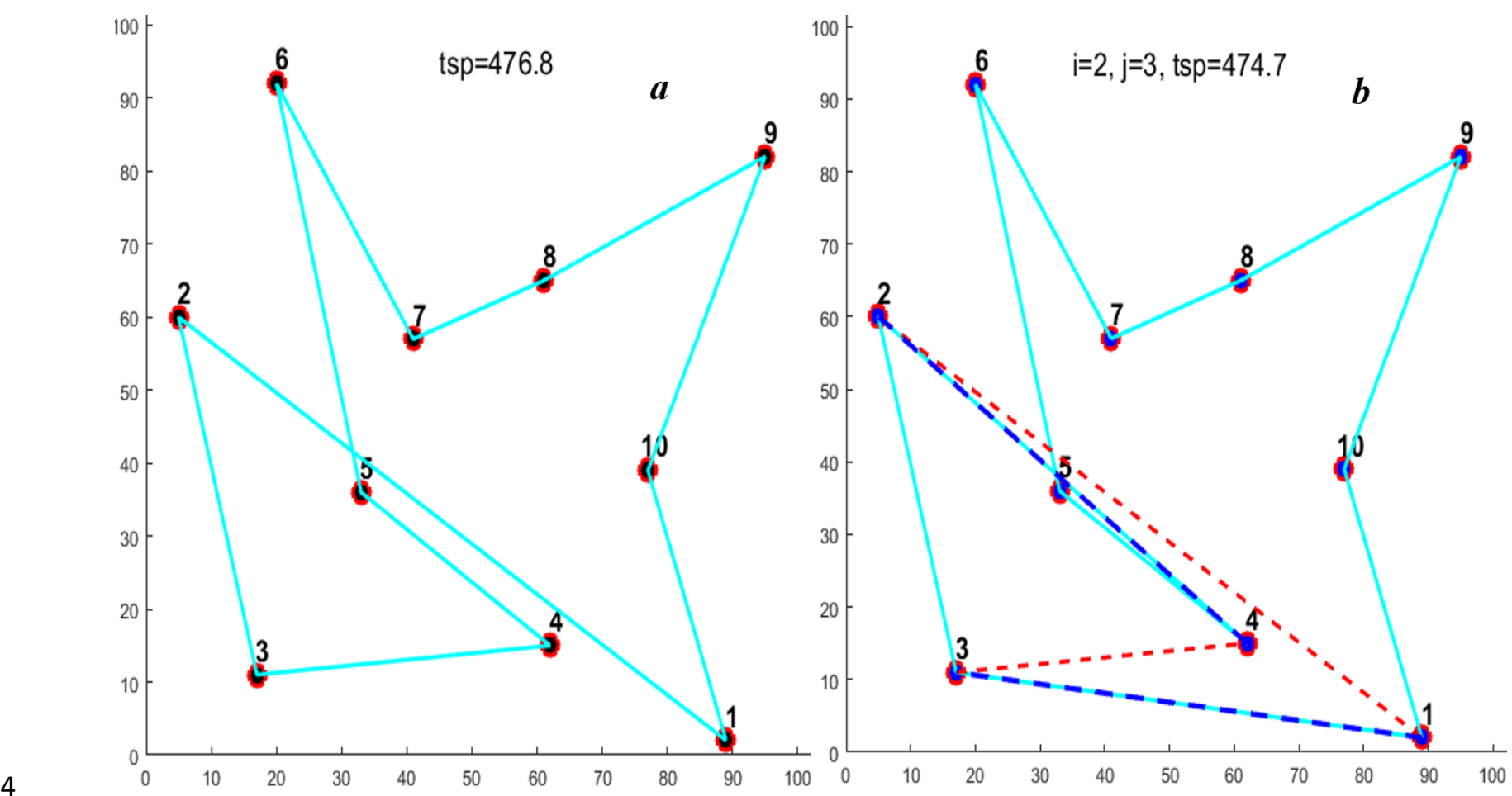

Fig 4 the original path of ten nodes (a) and moved $x_{3}$ to the front of $x_{2}$ (b)

if $d^{2} 2$ then involved six segments line (or edges) Fig.5a, three red dashed lines and three blue dashed lines, respectively. And the difference of tour length between the segments line should be as following:

$d=d_{i-1, j} \quad t_{j, i} \quad d+_{j, j 1+} d_{i-1, i-} d_{j \overline{1}_{j-j}} d_{j, j} \bar{i}_{+} \quad 2,3,=, n \quad 1, y \quad i-1, i \quad z \quad \quad+n$ (6)

As a show, if $d<0$, only exchange the adjoin nodes $x_{i}$ and $x_{j}$ or move $x_{j}$ to the front of $x_{i}$. When moving multiple nodes (from two to five) that should make a judge whether may insert forward or insert backward, and only picking the proper insert direction that can make $d$ a decrease. Depending on the orientations of the paths within the current structure, the path between $i$ and $j$ city may have to be reversed. As illustrated above that move could be forward or backward. For example, the original path (Fig.4a) that only by four times single node moving and instantly can achieve the optimum result as shown in Fig.5-Fig.6 show. But after another random permutation of the current tour that the move times will increase. Also, the move step always stops after may $\mathrm{n}$ iterations if its rerouting strategy fails to improve the current best solution and will immediately execute another step. A similar local search algorithm starts at some location in the search 

space and subsequently moves from the present location to a neighboring location or interval two and three nodes. Move algorithm is used for the purpose of improving tour arrangement operations.
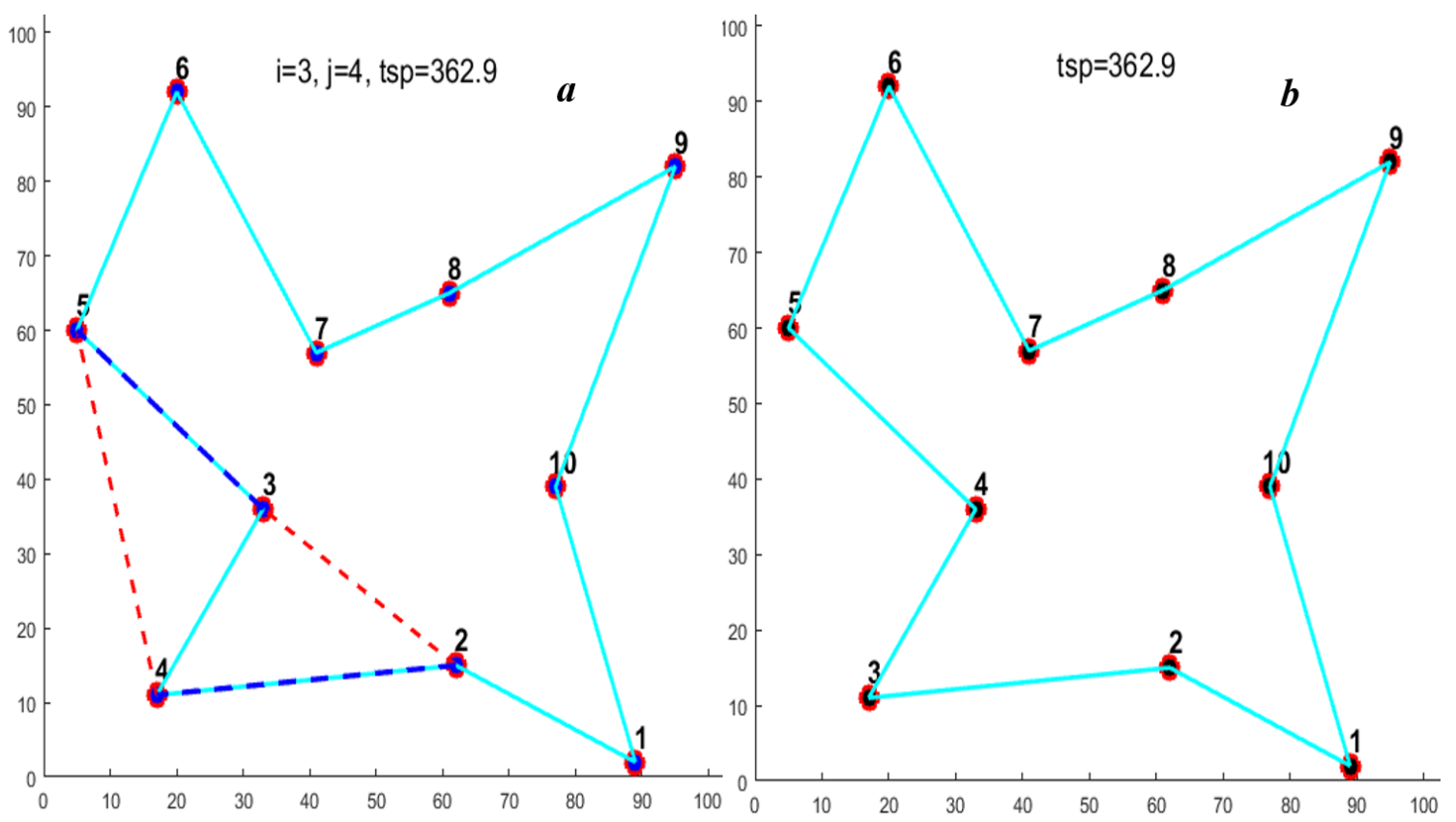

295

Fig 6 moved $x_{4}$ to the front of $x_{3}$ (a) and the optimal path of ten nodes problem (b)

Fig 5 moved $x_{4}$ to the front of $x_{2}(\mathrm{a})$ and $x_{5}$ to the front of $x_{3}(\mathrm{~b}$
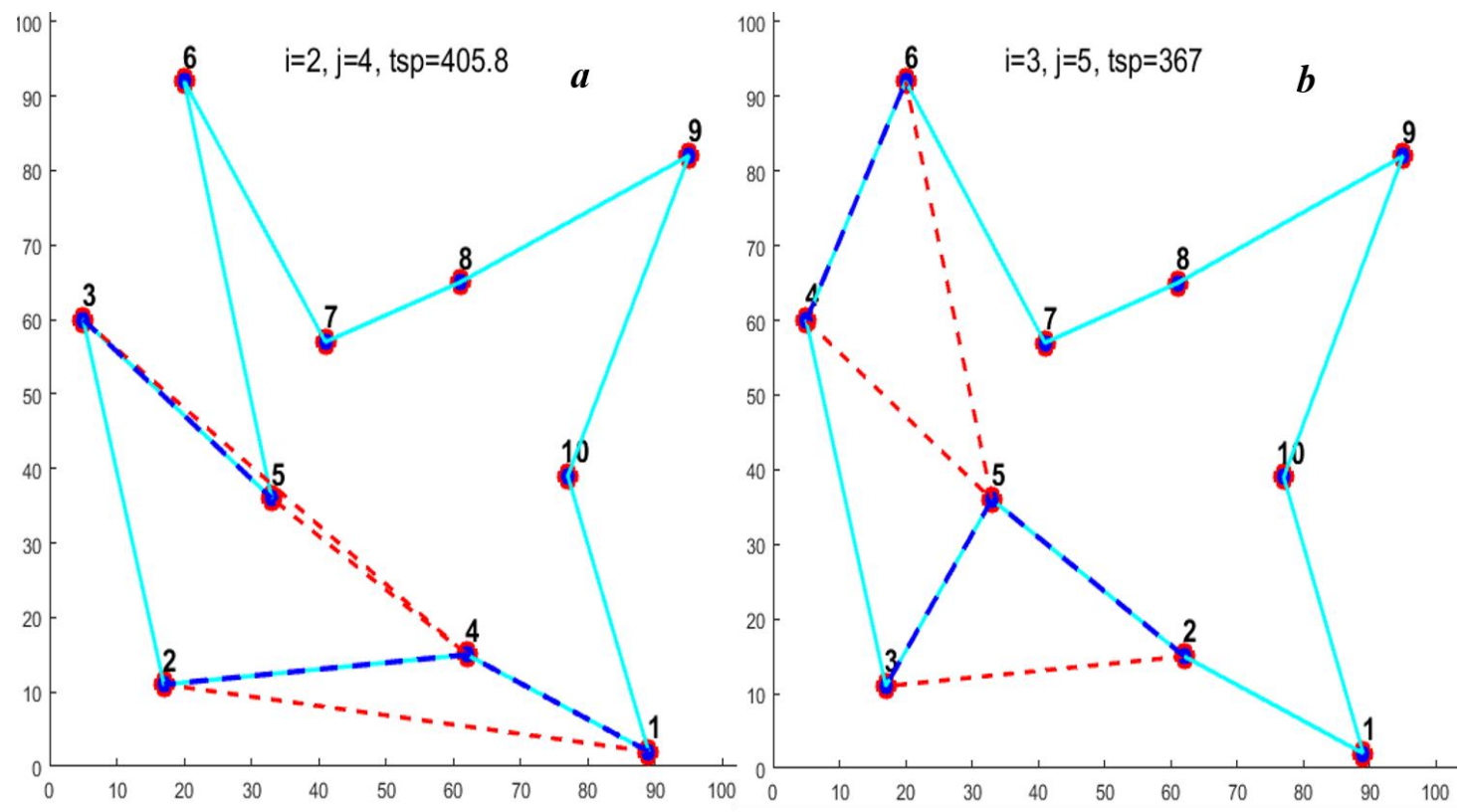
The concept of Flip in this paper that means Fliplr. If flip a segment line (or an edge) can make the $t s p(1)$ value decrease, then executed flip step operation. As Fig.7a, there is a crossover link between $x_{4}, x_{5}$, and $x_{6}$, and this kink between edge $\left(x_{3}, x_{4}\right)$ and edge $\left(x_{6}, x_{7}\right)$ make tours more complex and lengthy. Therefore, removing these kinks is wise action. Eliminating kinks from the current tour that means the path 3-4-5-6-7 should become 3-6-5-4-7, and achieve that only to flip $x_{4}, x_{5}, x_{6}$ to $x_{6}, x_{5}, x_{4}$. The difference of tour length between the new path and old path as following (Fig.7b, two blue and two red dashed lines): $d=d_{3,6} \quad t_{4,7} \quad d_{3,4} \quad d_{6,7} \quad 159 \cdot(7)$

As shown not only the $\operatorname{tsp}(1)$ value decrease by 15.9 , and also eliminated the crossover link. For another flip edge $\left(\mathrm{x}_{4}, \mathrm{x}_{7}\right)$ and edge $\left(x_{5}, x_{7}\right)$, and the red and blue dashed lines that were the change segments line 308 Fig.8a-b.
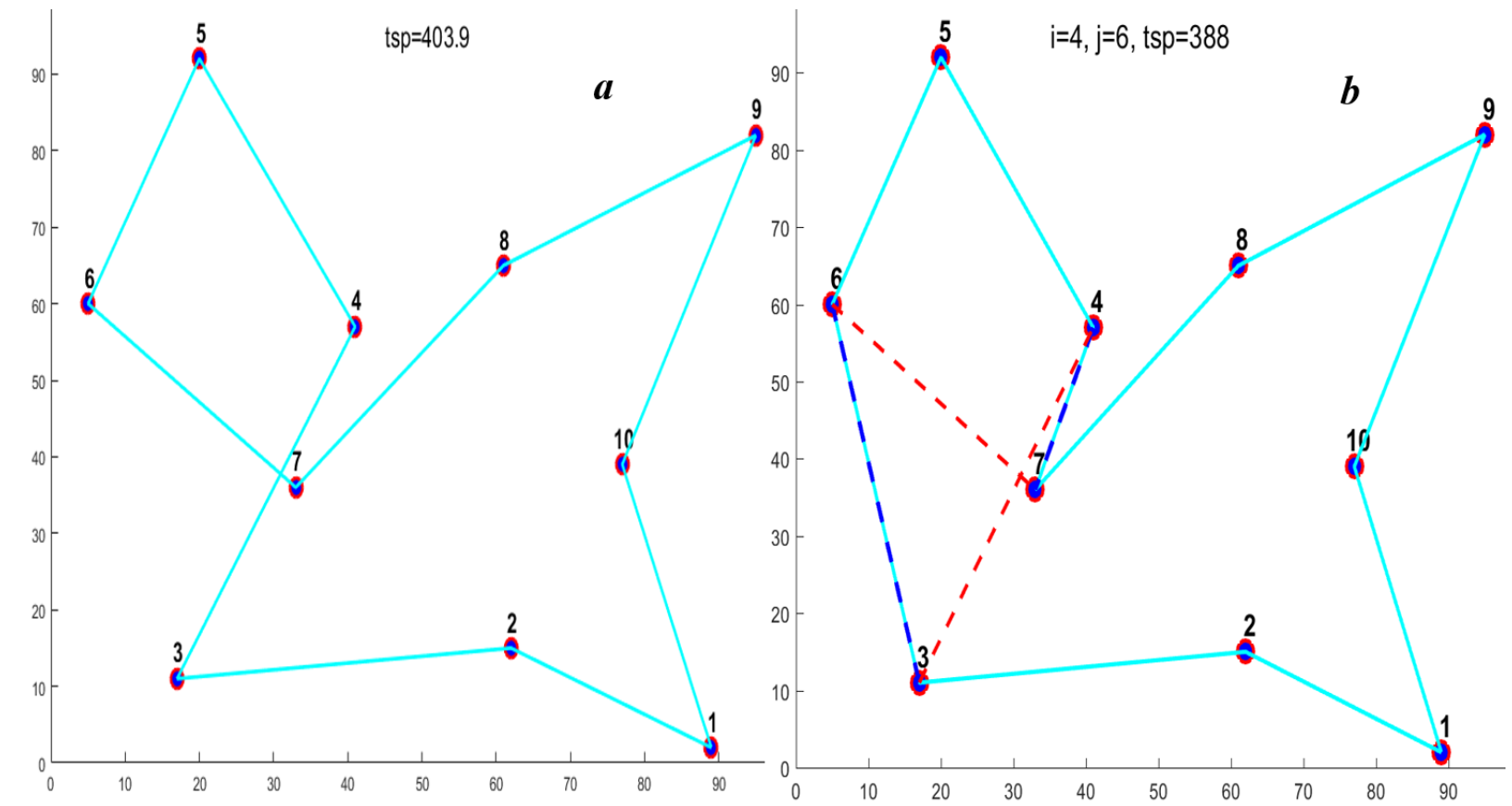

Fig 7 the original path ten nodes (a) and flip edge $\left(x_{4}, x_{6}\right)$ path (b) 


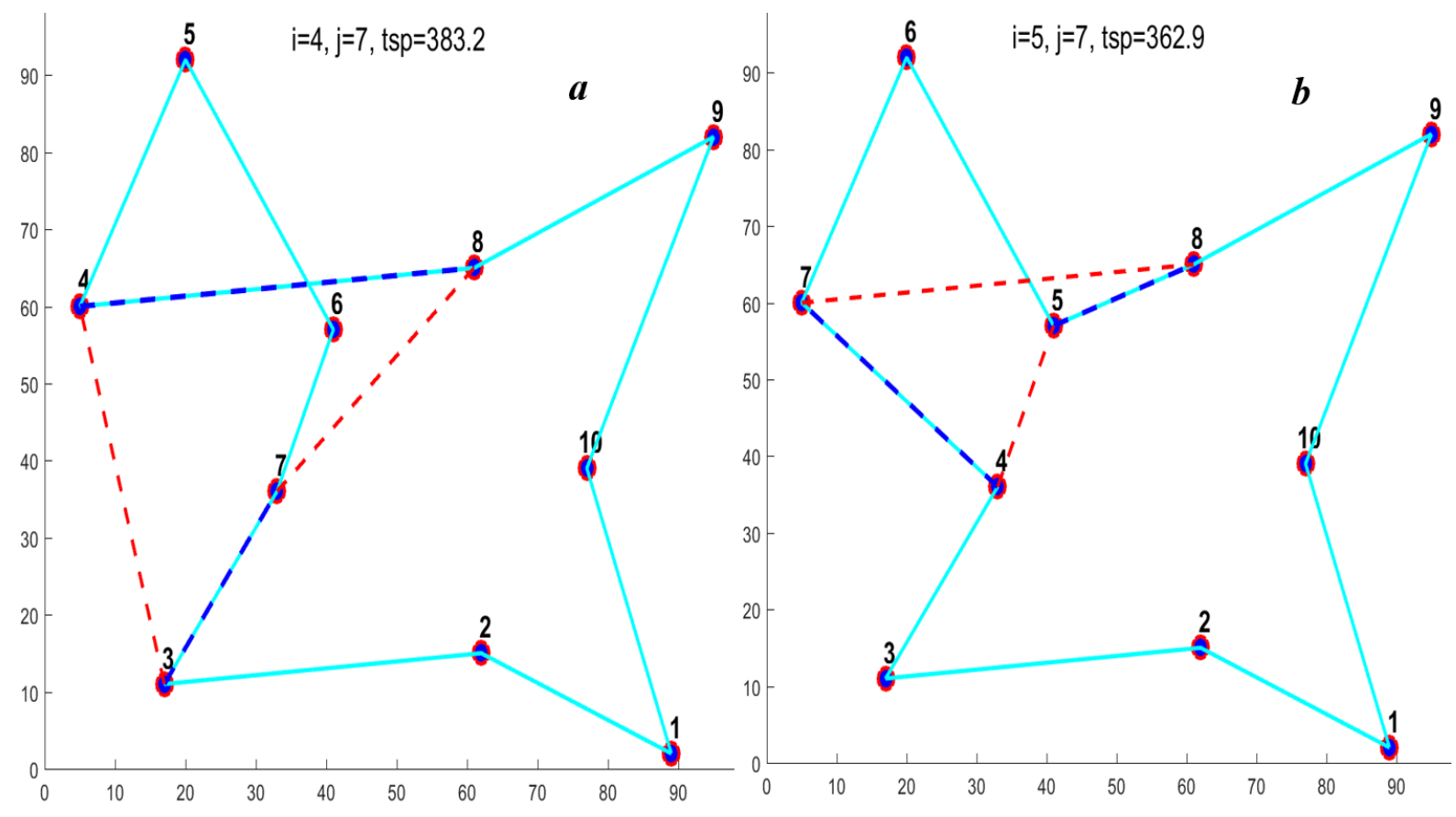

Fig 8 the path of flip edge( $(x 4, x 7)$ (a) and flip edge(x5, x7) (b)

Universally, before or after flip that the difference of tour length $\delta$, as follows, which only involved four segments line (or edges):

$$
d=d_{i-1, j} \quad \uplus_{i, j+} \quad d_{i 1 ; i} \quad d_{\overline{j, j} 1+} i \quad 2, \quad, n \gg j, j-i \quad 2, \dot{F}=3,+, n-
$$

Where should instantly flip edges $\left(x_{i}, x_{j}\right)$ path, when the $\delta<0$. As above example Fig.7a, which only flip three times and could realize the optimum result. If the original path was completely disarrayed, requiring number of times of flip will comfortably increase. As we know, flip step can easily eliminate the crossover link than others step hard completed. It is an implementation of the path transform that for not generated the kinks yet before, and also play a complementary role for combining the exchange and move steps, accelerating the speed that tends to optimum results and finds the best solution.

\section{Contraction-Expansion Algorithm}

It is easy to achieve global optimal for simple TSPs that only combined exchange, move and flip three steps, while is hard to achieve global optimization for generalized traveling salesman problems. Here, we used the contraction-expansion algorithm to expand the search spaces for searching for the best tours. The algorithm is specified in exchanges (or moves, flips) that can convert one candidate solution into another gainful move. Therefore, we set of rules for $\delta$ that gave an appropriate critical value ( $c r t$ supplement algorithms 11), if $d \$ c r t$, even though exchange and move steps may increase target function $t s p(1)$ value, we are still forced to execute these operations (the process of operation that we call redeploy of 
enforceability). It can help to drop out the pitfall of the local optimal, and greatly increase the possibility of realizing global optimal.

With the feasibility criterion that the loop is stopped as soon as the chain represents a gainful exchange, and move, or when the chain cannot be produced better optimum tsp (1) value (no longer less than $t s p$ value) anymore. In theory, there will be up to $\mathrm{n}$ iterations in the loop, where $\mathrm{n}$ is the number of nodes. In practice, however, the number of iterations is much smaller (due to the confirm of critical value). So we set rules for the critical value. if $c r t$ is too small, it cannot drop out the pitfall of local optimum. And if $c r t$ is too large that should not have exchanged and moved, it's would be forced to implement, and after one loop or more loops that the sequence of the tour would be disarray and have a strong impact on the effectiveness of implementation. Meanwhile, as the change of $c r t$ with the search loops alters, it appeared the form of the wave for changing. Therefore, we spent plenty of time researching and seeking the best function that can reasonably adjust and control the crt changing. Here, we found the function (9) that was a negative exponent function, which was used to the method of feedback regulation. This stop criterion (a product the feasibility critical value) plays a major role in the efficiency of the algorithm.

$c r t=c r t * e^{(-0)}$

\section{E. Combinatorial Optimization of Program Elements}

Pseudocode of those programs that included exchange, move and flip (involved one node and from two to five nodes choose together), besides, combined the contraction-expansion algorithm and process of redeploying of enforceability which compiled more than twenty program elements. And the implement of the sequence of those programs that is the core of high effectiveness achieve global optimal. The implement of rules that are after exchange forward following on exchange backward with the different types of program elements, also, such that avoid an ineffective exchange of the nodes which should be legitimately configuration exchange between the one node and multiple nodes. A large computational experiment show that reasonably arrange the sequence of program elements is the point to increase the power of achieving the global optimum for traveling salesman problems. As far, that combinatorial optimization of program elements still not the best and yet have the space to reach the optimal.

Practically, after certainly loops that random to implement those programs of elements, it can prevent happened of invalid remove and vibration from the process of exchange and move nodes, also, yielding a better tour solution and more efficient to nearness optimal solution. Included all process of these program of elements is called loop times (round). It is impossible to achieve the global optimal for complicated traveling salesman problems just one loop times, and even hundreds of cycle rounds for large scare TSPs. But for small or middle scare TSPs that above procedure can produce a better tour solution. 
372 Above that procedure of exchange, move and flip the position of the start point, and endpoint won't 373 implement the exchange and move steps. Also, it was hard to generate efficient exchange for the closely 374 linked nodes. Sometimes, it will be not only left the dead corner to achieving the optimal tour, and also 375 obstruct from yield the global optimal tour. Therefore, after certainly loop times that need to redefine the 376 start node and keep the search for the best optimal tour. Usually, it is based on before generating the best 377 tour that random forward or backward move to certainly nodes, and rotate TSP cycle that the lots of 378 position as the new start(end) point. By doing this made the procedure of exchange, move and flip more 379 unobstructed (no dead corner) for the complicate TSPs and increase the speed of the process of search the 380 best tour solution.

\section{EXPERIMENTAL STUDY}

In this section, we describe the test problems and implementation details of the algorithms that we use in our computational study. We present and discuss the results generated by other different algorithms that have reported from the literature on the same problems in TSPLIB. Also, in our experiments, which we 389 presented a new evaluation index described as following, and it was used to evaluate the performance of 391 EMF-CE algorithm. And used the following index that was described as the efficiency index of EMF-CE algorithm.

\section{A.Evaluation Index}

To test developed operator performance, which test problems for small and medium scale (51-1000 cities) TSPs were taken from the TSPLIB online library[67] and the website at (http://www.iwr.uniheidelberg.de/groups/comopt/software/TSPLIB95/. As we all know, if an algorithm can reach the nearby global optima for all kinds of traveling salesman problems, then it should be the best program or software. For all the test problems, we both random permutation for the initial path (disorganize the original data sequence) as the restart path after yield optimal solution. Which also records the optimal rates and time of achieved the global optima twenty times. And gave the optimal solution of the result was from the Condore 1.1(current best software) running in windows as the benchmark. 
We used the rates of optimal solution $\left(R_{o}=N_{\delta} N_{r}, N_{o}\right.$ is the number of optimal, $N_{r}$ is the number of runs), the average tour length, and median tour length as the power of evaluation index. And the efficiency of the index included the shortest time, average time, and median time. By reason of complicated problems in a set of loops can't all achieve global optima and early quit, which also can't calculate the meantime to reach to global optima. In some way, it was fake that if we take early quit time into consideration. While median time (must have more than half to achieve global optima) isn't affected by extremes value; therefore, it is more stable and more valuable as an efficiency index.

The first two sets of experiments were eil51.tsp and ch150.tsp two small scale problems were used to test the executable of CPU time and rates of optimal. For random permutation the initial path as start path, and instantly record the CPU time as long as obtained the optimal solution. The CPU time-frequency distribution resulted from multiple replications executes. In early, obtained a few times for executing, while the CPU time was unimodal skewed normal distribution which was incompatible with the theory. But with the continuous running 1000 times, its CPU time of frequency distribution was similar to the formerly cardinal principle and showed the extremely skewed distribution as follows Fig.9.

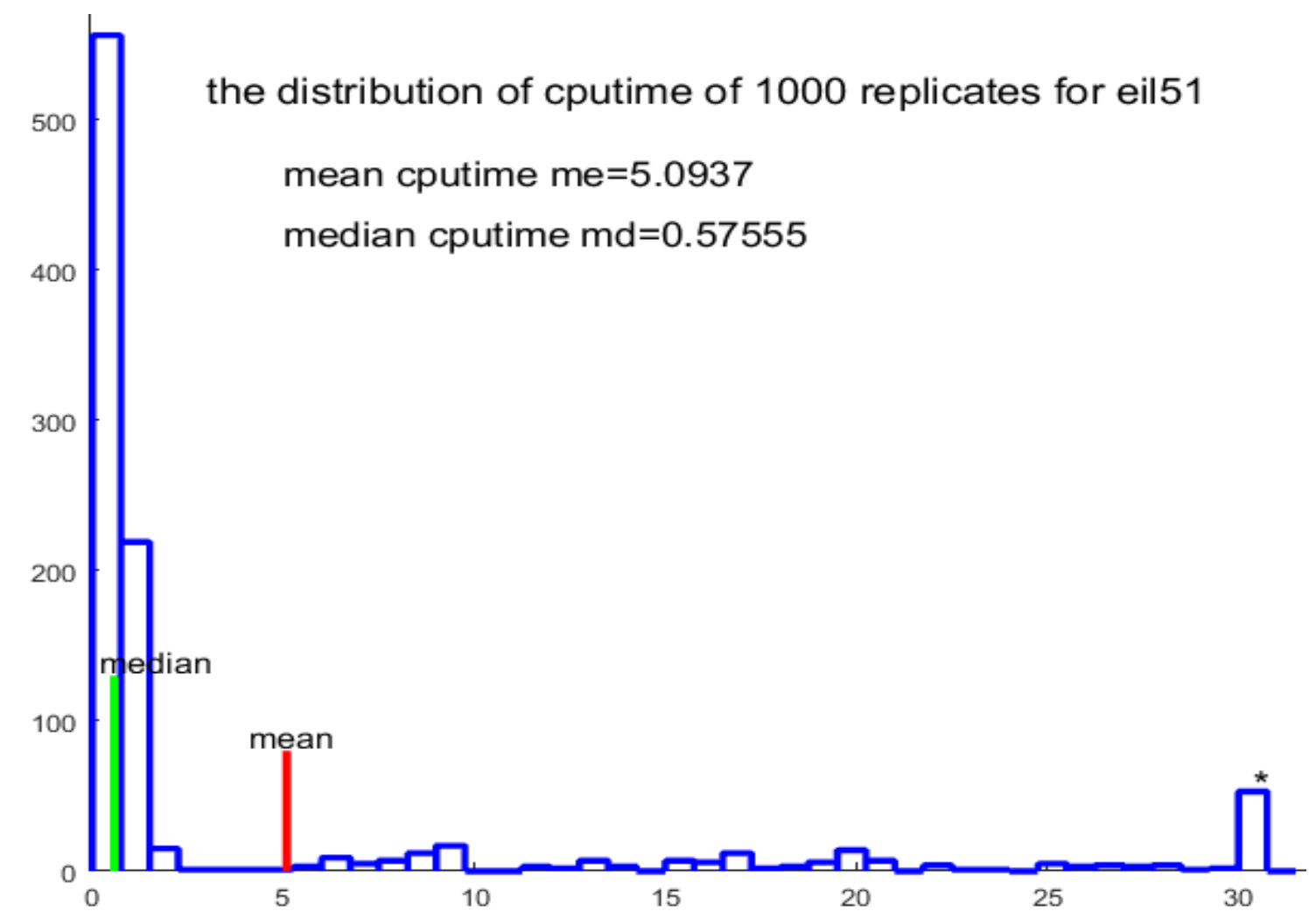

Fig 9 the frequency distribution of CPU time with 1000 replicates for eil51.tsp problem

Executed one thousand times for this issue and the rates of optimal was $100 \%(R o=1000 / 1000)$, also all of the lengths of the tour were 426 , but between all of them, the CPU time has a more significant 
422

423

424

425

426

427

428

429

430

431

432

433

434

435

difference Fig.9. As the show that the last group was 53 times for CPU time more than 30 seconds. The minimum and maximum CPU time was $0.05 \mathrm{~s}$ and 65.01s, respectively. Because exist of the extreme value, the mean of the CPU time was 5.09s (the red line show) which wasn't reasonable as the efficiency of the index for indicating the effectiveness of the algorithm. While the median was $1.61 \mathrm{~s}$ (the green line show) and has run for 775 times that the CPU time was less than 3s to achieve global optimal, also, it is indicating the median better than average as the efficiency of index.

As we all know, the tour length of the global optimal of eil51.tsp problem was 426. While we dig deeper and found that situation was different. We tried to retain 3 decimals, the tour length was 429.118, but this is not the real global optimal, and the tour length of global optimal should be 428.871 (the rounded number was 427) Fig.10-b. Otherwise, if round the tour length to nearest the integer was 426 Fig.9, keep integer distance of between $d_{25,26}+d_{26,27} \quad t_{27,28}$ and $d_{25,27}+d_{27,26} \quad t_{26,28}$, there are not the only one optimal tours. There are groups of test problems from TSPLIB that existed retain integer for the tour length will be influenced to produce optimal results. While for a large data TSP problem, the integer has little effect

on
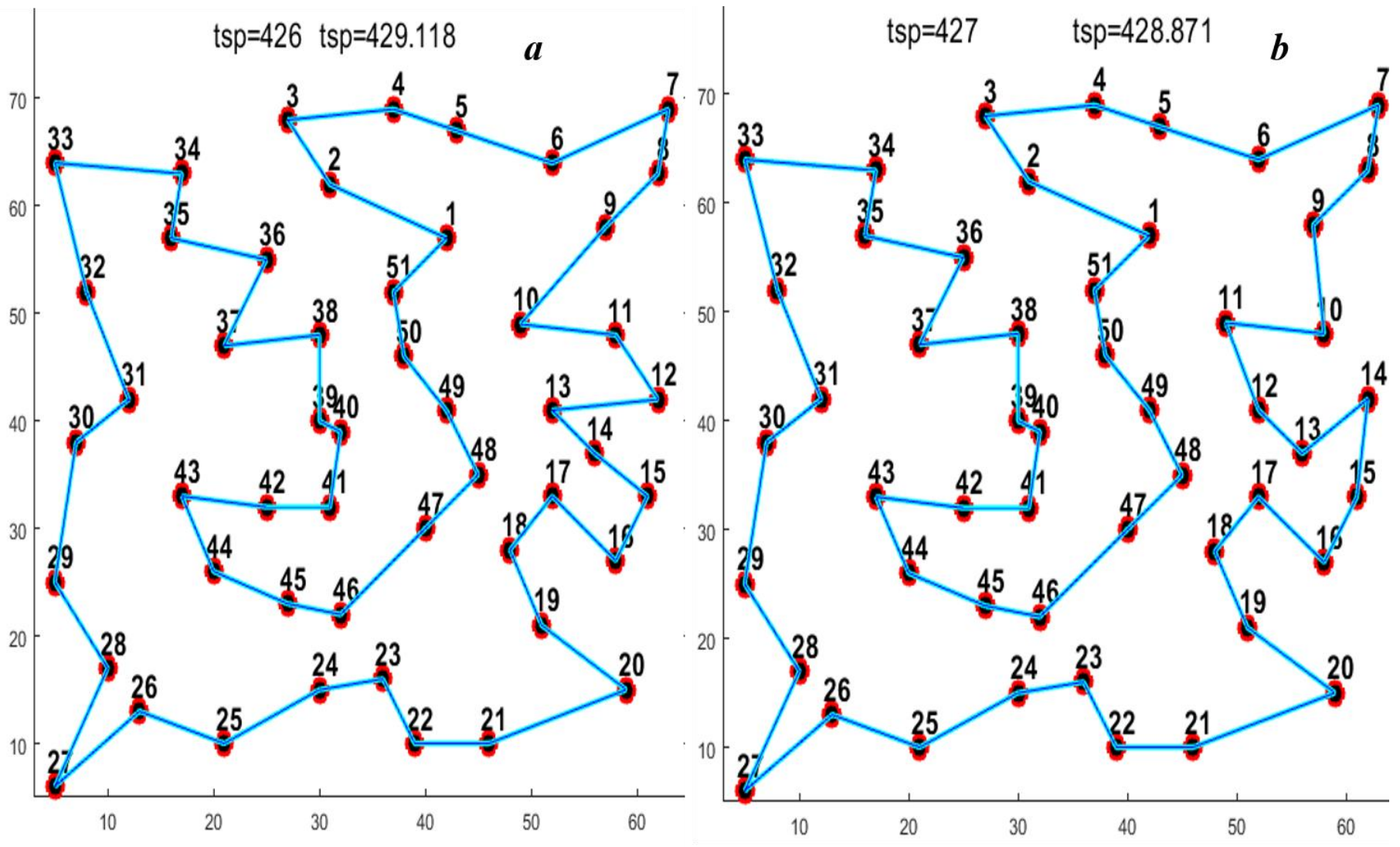

Fig 10 the global optimal solution of integer (a) and non-integer (b) for eil51.tsp problem 
the optimal results and relatively easy to obtain the optimal results, also convenient to compare each other with different algorithms, which maybe was the reason for the widespread use. But there are some test TSP problems, if retain 4 6 decimals, the optimal results will be the global optimal.

Where also executed one thousand times for ch150.tsp issue and the rates of optimal was $90.5 \%(\mathrm{Ro}=905 / 1000)$, all of the lengths of the tours were 426 , but between all of them, the CPU time has a more significant difference Fig.10. And have 156 times for the CPU time more than 60s in the last group as show Fig.10. The results indicated that minimum, average, maximum, and median tour length was 6528 (integer), 6593, 6531.06, and 6528, respectively. Also, the minimum, average, maximum, and median time were $1.04 \mathrm{~s}, 58.12,760.66$, and $1.61 \mathrm{~s}$, respectively. Indicating that already have Ro=726/1000 for the CPU time less than 3s. It was also indicating that median time or tour length will be better than average time or tour length as an index for proving the effectiveness of the algorithm. But the set of iteration times less than 300 that can't achieve global optimal.

There were three weak peaks that respectively nearby the 20s, 40s, and 50s both achieved the global optimal. After researched the program and found that appeared these phenomena, which has certain relations with the periodic variation of $\mathrm{crt}$. While these peaks relatively the crt value both in the bottom and easy to achieve global optimal after early big crt value broke the pitfall of local optimal. For the other test problems that the distribution of CPU time both corresponding to the first two problems. This indicates

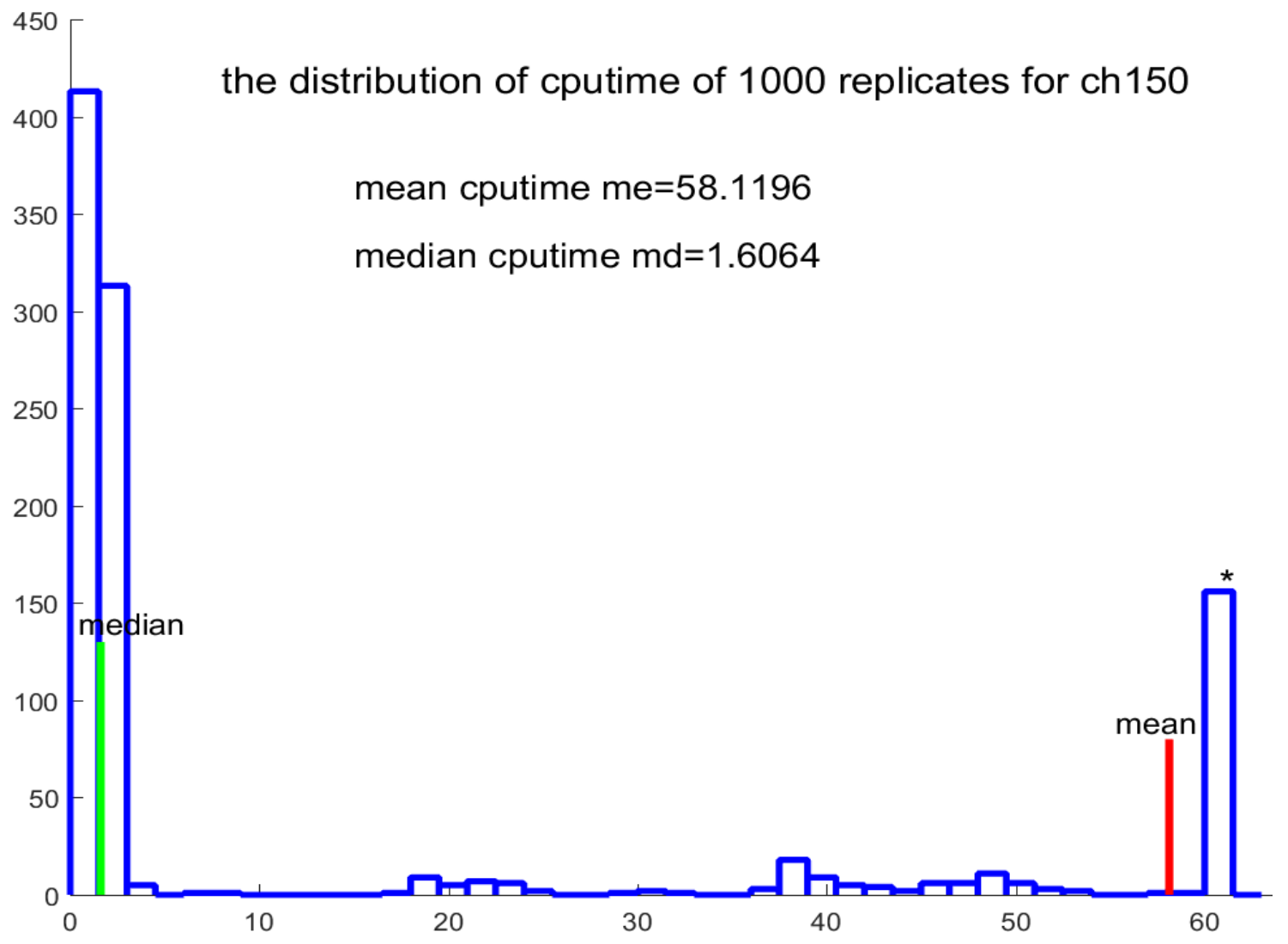

Fig 10 the frequency distribution of CPU time with 1000 replicates for ch150.tsp 


\section{2}

that the new algorithm to achieve global optimal with a shorter median time, and another small part of it takes a long time to run. With the complexity of the problem, this phenomenon will become more obvious. This also reflects algorithmic programs to improve the possibility of need.

\section{B. Implementation Details}

All the experimental results were obtained by an Intel(R) Core(TM)2 Duo $2.93 \mathrm{GHz}$ with 2-GB main memory using MATLAB environment and running the Windows 7 operating system. For comparing EMF-CE with other algorithms in terms of CPU time, we scaled the CPU time of each algorithm by an appropriate scaling coefficient related to its processing system. We utilized the results reported to obtain the scaling coefficients as shown in Table I. Note that the codes made by $3-\mathrm{GL}$ programming languages such as $\mathrm{C} / \mathrm{C}++$, PASCAL, and FORTRAN are more efficient than M-codes in the MATLAB interpreter. Nevertheless, we did not consider any scaling coefficient for comparing M-codes with 3-GL codes. In other words, it is expected to obtain better performance by implementing our algorithm using an efficient programming language like $\mathrm{C}$ or $\mathrm{C}++$.

Table I

SCALING COEFFICIENTS FOR COUNTERPART ALGORITHMS

\begin{tabular}{ccccc}
\hline \multirow{2}{*}{ Counterpart Algorithm } & \multirow{2}{*}{$\begin{array}{c}\text { Processing } \\
\text { System }\end{array}$} & $\begin{array}{c}\text { Program } \\
\text { Language }\end{array}$ & $\begin{array}{c}\text { Scaling Coefficients } \\
\text { Prog.Lan }\end{array}$ & MATLAB \\
\hline ASA-GS[26] & $2.8 \mathrm{GHz}$ & $\mathrm{C}++$ & 1.00 & $>1.00$ \\
CONN[62] & $1.4 \mathrm{GHz}$ & $\mathrm{C}++$ & 0.49 & $>0.49$ \\
eISOM, ESOM, Budinich[19] & $1.4 \mathrm{GHz}$ & $\mathrm{C}++$ & 0.48 & $>0.48$ \\
RABNET-TSP[36] & $3.0 \mathrm{GHz}$ & MATLAB & 1.06 & $>1.06$ \\
GCGA[68] & $2.8 \mathrm{GHz}$ & C++ & 0.99 & $>0.99$ \\
HGA[69] & $2.3 \mathrm{GHz}$ & $\mathrm{C}++$ & NA & NA \\
GSTM[70] & $2.8 \mathrm{GHz}$ & Delphi & NA & NA \\
ACS[16] & $200 \mathrm{MHz}$ & C++ & NA & NA \\
SA[46] & SPARCULTRA5 & C++ & NA & NA \\
PSO-ACO-3Opt[38] & $2.8 \mathrm{GHz}$ & $\mathrm{C}++$ & NA & NA \\
\hline
\end{tabular}

The percent differences (relative error) have been computed using:

$$
R E(\%)=\frac{l-l_{\text {opt }}}{l_{\text {opt }}} 3100 \%
$$

Where $l$ were best tour lengths (BL, the result from EMF-CE), average tour lengths(AL), or median tour lengths(ML), however, $l_{\text {opt }}$ is the optimal tour lengths(OPT). Note that for each TSP EMF-CE gives the same solution in all runs. 
We select 43 problems from the TSPLIB online library. Each problem is randomly generated. We use Euclidean distances (all of the Euclidean distances are real distances). The problems range in size from small to middle-scale 51 to 1000. Following Johnson and McGeoch[71], and only one running pr2392 was performed for this instance, which were used in previous computational studies (see Jin et al. $[19,25,26,62,70]$. Each entry gives the number of nodes in the problem (e.g., eil51 has 51 nodes). We use the approximate Euclidean distance metric specified in TSPLIB. Moreover, we did not apply EMF-CE to the instances with 3000 or more cities since it would have taken too much time (future work for large TSP). Therefore, we considered only a qualitative comparison between the different algorithms. The results are listed in Table II. For each benchmark problem, the first ten columns of this table are 1) number; 2) the TSP name; 3) optimal tour length (OPT) as reported in TSPLIB, and some instances renew calculated that used the Concorde (http://www.math.uwaterloo.ca/tsp/concorde/gui/gui.htm), and 4)described in V.A 5) were Shortest tour lengths (SL, the result from EMF-CE) and RE(\%); 6) average tour lengths(AL) and RE(\%); 7) median tour lengths(ML) and $\mathrm{RE}(\%)$; 8) the shortest of running time in second; 9) the average of running time in second; 9) the median of running time in second. And the CPU time of average and median distribution as the Fig.11 scatter diagram demo. Also, in order to facilitate comparison with different algorithms. According to Pepper et al. [46], we split the set of selection problems into the first three sets that 51 to 299 nodes(31 instances), 318 to 574 nodes(7 instances), and 666 to 100 and one 2392 nodes(5instances).

\section{TABLE II}

EMF-CE SOLUTIONS TO 43 BENCHMARK TSP FROM TSPLIB

\begin{tabular}{cccccccccc}
\hline No. & Instances & OPT & Ro & SL(\%) & AL(\%) & ML(\%) & ST(s) & AT(s) & MT(s) \\
\hline 1 & ei151 & 426 & $20 / 20$ & Optimal & $426(0)$ & $426(0)$ & 0.21 & 20.71 & 12.95 \\
2 & st70 & 675 & $20 / 20$ & Optimal & $675(0)$ & $675(0)$ & 0.52 & 15.98 & 13.37 \\
3 & eil76 & 538 & $20 / 20$ & Optimal & $538(0)$ & $538(0)$ & 1.09 & 75.96 & 66.14 \\
4 & pr76 & 108159 & $20 / 20$ & Optimal & $108159(0)$ & $108159(0)$ & 12.85 & 32.62 & 29.37 \\
5 & rat99 & 1211 & $20 / 20$ & Optimal & $1211(0)$ & $1211(0)$ & 2.33 & 77.97 & 73.00 \\
6 & kroA100 & 21282 & $20 / 20$ & Optimal & $21282(0)$ & $21282(0)$ & 0.67 & 39.54 & 21.37 \\
7 & kroB100 & 22141 & $20 / 20$ & Optimal & $22141(0)$ & $22141(0)$ & 7.88 & 150.29 & 156.76 \\
8 & kroC100 & 20749 & $20 / 20$ & Optimal & $20749(0)$ & $20749(0)$ & 2.20 & 43.71 & 27.62 \\
9 & kroD100 & 21294 & $20 / 20$ & Optimal & $21294(0)$ & $21294(0)$ & 1.00 & 37.05 & 24.24 \\
10 & kroE100 & 22068 & $7 / 20$ & Optimal & $22097.3(0.13)$ & $22100(0.15)$ & 32.11 & 1287.12 & 182.31 \\
11 & rd100 & 7910 & $20 / 20$ & Optimal & $7910(0)$ & $7910(0)$ & 16.26 & 149.86 & 159.14 \\
12 & eil101 & 629 & $14 / 20$ & Optimal & $630.35(0.21)$ & $629(0)$ & 24.83 & 173.40 & 173.51 \\
13 & in105 & 14379 & $20 / 20$ & Optimal & $14379(0)$ & $14379(0)$ & 16.92 & 37.33 & 26.66 \\
14 & pr107 & 44303 & $20 / 20$ & Optimal & $44303(0)$ & $44303(0)$ & 0.36 & 8.86 & 3.75 \\
15 & pr124 & 59030 & $20 / 20$ & Optimal & $59030(0)$ & $59030(0)$ & 6.09 & 70.23 & 50.38 \\
16 & bier127 & 118282 & $3 / 20$ & Optimal & $118483.4(0.17)$ & $118476.7(0.16)$ & 101.83 & 396.94 & 388.89 \\
17 & ch130 & 6110 & $7 / 20$ & Optimal & $6130.45(0.33)$ & $6128(0.29)$ & 149.25 & 337.28 & 335.73 \\
18 & gr137 & 697 & $20 / 20$ & Optimal & $697(0)$ & $697(0)$ & 2.02 & 166.41 & 172.51 \\
19 & pr144 & 58537 & $20 / 20$ & Optimal & $58537(0)$ & $58537(0)$ & 4.68 & 118.55 & 96.50
\end{tabular}




\begin{tabular}{|c|c|c|c|c|c|c|c|c|c|}
\hline 20 & $\operatorname{ch} 150$ & 6528 & $18 / 20$ & Optimal & $6529.71(0.03)$ & $6528(0)$ & 1.80 & 57.99 & 1.90 \\
\hline 21 & kroA150 & 26524 & $8 / 20$ & Optimal & $26578.8(0.21)$ & $26566(0.16)$ & 204.79 & 429.27 & 346.90 \\
\hline 22 & kroB150 & 26130 & $5 / 20$ & Optimal & $26166.6(0.14)$ & $26150(0.08)$ & 306.43 & 1291.90 & 1350.45 \\
\hline 23 & pr152 & 73682 & $20 / 20$ & Optimal & $73709.2(0.04)$ & $73682(0)$ & 0.89 & 114.19 & 84.65 \\
\hline 24 & u159 & 42080 & $18 / 20$ & Optimal & 42104.4(0.06) & $42080(0)$ & 1.90 & 106.94 & 41.01 \\
\hline 25 & kroA200 & 29368 & $1 / 20$ & Optimal & $29543.4345(0.6)$ & $29545.5(0.6)$ & 532.67 & 2088.46 & 861.85 \\
\hline 26 & kroB200 & 29437 & $0 / 20$ & $29440(0.01)$ & 29597.7(0.55) & $29530.5(0.32)$ & 628.99 & 2622.08 & 2181.19 \\
\hline 27 & ts 225 & 126643 & $20 / 20$ & Optimal & $126643(0)$ & $126643(0)$ & 119.99 & 399.69 & 377.58 \\
\hline 28 & pr226 & 80369 & $2 / 20$ & Optimal & $80378.5(0.01)$ & $80373(0)$ & 1592.06 & 2737.44 & 2434.60 \\
\hline 29 & pr264 & 49135 & $12 / 20$ & Optimal & 49155.95(0.04) & 49135(0) & 260.69 & 864.18 & 857.53 \\
\hline 30 & $\mathrm{a} 280$ & 2579 & $8 / 20$ & Optimal & $2585.2(0.24)$ & $2590(0.43)$ & 988.79 & 1746.69 & 1601.42 \\
\hline 31 & pr299 & 48191 & $0 / 20$ & $48226(0.07)$ & $48418.9(0.47)$ & $48435.5(0.51)$ & 188.22 & 4633.67 & 2082.20 \\
\hline 32 & $\operatorname{lin} 318$ & 42029 & $0 / 10$ & $52152.99(0.30)$ & $42152.99(0.3)$ & $42152.99(0.3)$ & 7.30 & 225.98 & 9.36 \\
\hline 33 & $\operatorname{rd} 400$ & 15281 & $0 / 10$ & $15338.60(0.40)$ & $15480.174(1.3)$ & 15475.61(1.27) & 626.35 & 28878.17 & 31025.00 \\
\hline 34 & f1417 & 11861 & $0 / 10$ & $11873(0.10)$ & $11882.3(0.18)$ & 11882(0.18) & 6761.00 & 14498.14 & 14801.00 \\
\hline 35 & pr439 & 107217 & $0 / 10$ & $107267(0.05)$ & $107985.5(0.72)$ & $107941(0.68)$ & 8343.34 & 24983.92 & 30897.71 \\
\hline 36 & pcb442 & 50778 & $0 / 10$ & $51009.68(0.46)$ & $51009.68(0.46)$ & $51009.68(0.46)$ & 17.58 & 1293.36 & 152.25 \\
\hline 37 & att532 & 86729 & $0 / 10$ & $86779(0.06)$ & $88232.17(1.73)$ & $88145(1.63)$ & 30961.84 & 42216.81 & 39462.64 \\
\hline 38 & u574 & 36905 & $0 / 10$ & $36955(0.14)$ & $37662.67(2.05)$ & $37819(2.48)$ & 43611.95 & 77116.72 & 74097.63 \\
\hline 39 & gr666 & 3062 & $0 / 5$ & $3067.09(0.17)$ & $3098.836(1.2)$ & $3112(1.63)$ & 68419.00 & 136507.80 & 145240.00 \\
\hline 40 & u724 & 41910 & $0 / 5$ & $41960(0.12)$ & $42744.8(1.99)$ & $42840(2.22)$ & 136850.00 & 169554.00 & 145740.00 \\
\hline 41 & rat783 & 8806 & $0 / 5$ & $8846(0.45)$ & $9007.115(2.28)$ & $9033.23(2.58)$ & 194695.35 & 227887.42 & 225529.61 \\
\hline 42 & $\begin{array}{c}\text { dsj1000 } \\
\text { (CEIL2D) }\end{array}$ & 18660188 & $0 / 5$ & $\begin{array}{l}18660238 \\
(0.00027)\end{array}$ & $\begin{array}{c}18942217.2 \\
(1.51)\end{array}$ & $\begin{array}{c}19020000 \\
(1.93)\end{array}$ & 313540.00 & 344652.20 & 347430.00 \\
\hline 43 & pr2392 & 378032 & $0 / 1$ & $\begin{array}{c}378062.83 \\
(0.0082)\end{array}$ & $\begin{array}{c}378062.83 \\
(0.0082)\end{array}$ & $\begin{array}{c}378062.83 \\
(0.0082)\end{array}$ & 434484.00 & 434484.00 & 434484.00 \\
\hline
\end{tabular}

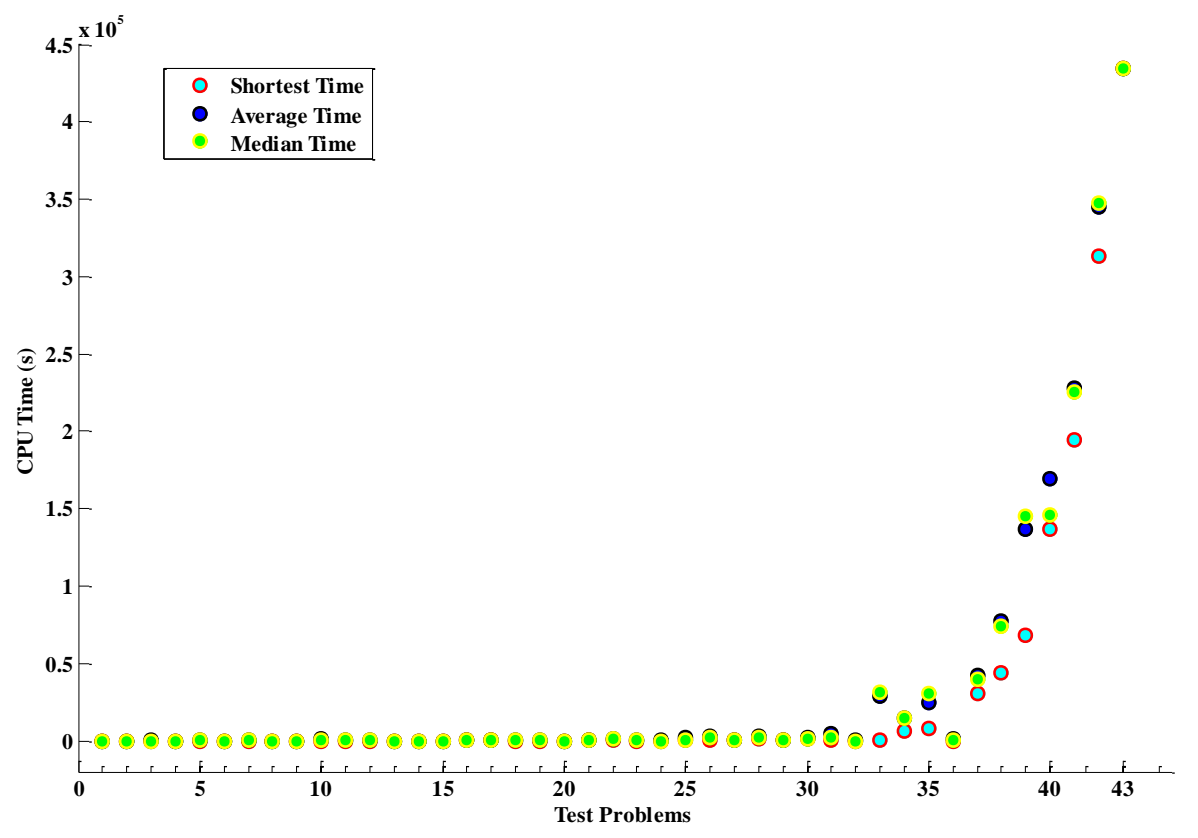


Fig. 11 shows the EMF-CE CPU time versus the number of cities for 43 benchmark TSPs. Fig. 7 shows the CONN CPU time versus the number of cities for all benchmark TSPs. In this figure, the CPU time versus $\mathrm{n}$ has been 504 fitted to a polynomial.

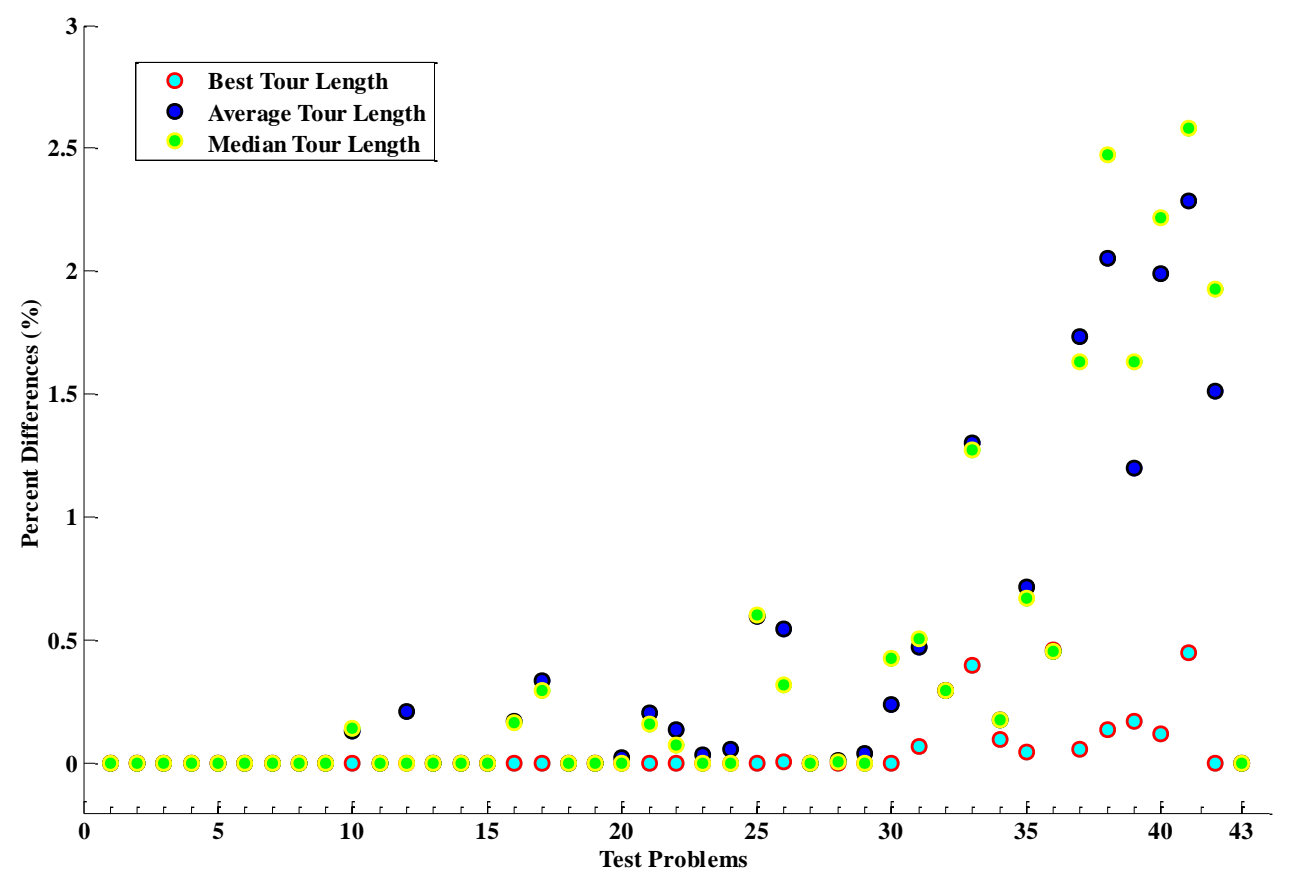

Fig.12 EMF-CE percent difference versus the number of cities for 43 benchmark TSPs from TSPLIB

As illustrated in Fig. 12, the three evaluation indexes showed that the solution qualities of EMF-CE are range $0.00 \% \sim 0.46 \%, 0.00 \% \sim 2.28 \%$, and $0.00 \% \sim 2.58 \%$ for all test benchmark TSPs TABLE II. Furthermore, EMF-CE gave the optimal solutions for 29 benchmark TSPs, in which the number of cities less than 300 .

\section{Comparing with Computationally Comparable Algorithms}

In this section, we compared EMF-CE with several well-known evolutionary algorithms, simulated annealing, evolutionary algorithms, and neural network including ASA-GS, GCGA, HGA, CONN, and LBSA, and so on. Which were reported in [26], [68], [69],[62] and [54], respectively. Also, these algorithm methodologies that we already briefly described in section II. EMF-CE has a competitive computational complexity with respect to these algorithms $\left(O\left(n^{3}\right)\right)$. The third set of experiments was performed on 38 benchmark TSPs from TSPLIB with 51(smallscale) to 783 cities (medium-scale). The results are only given the percent difference of shortest tour lengths in Table III.

For average shortest tour length that corresponding to the same TSP instances and EMF-CE provided 0.35\%, $1.49 \%, 0.82,3.11 \%$, and $0.02 \%$ improvements over ASA-GS, GCGA, HGA, CONN, and LBSA, respectively. When we also split the set of problems into three sets, as described in section V.C. Over the corresponding to first set problems Fig.13, we see that EMF-CE and LBSA was the most accurate algorithm with the difference of $0.003 \%$. However, EMF-CE provided 0.22\%, 0.92\%, 0.47\%, and 2.75\% improvements over ASA-GS, GCGA, HGA and CONN to the average shortest tour length, respectively; also, corresponding to second set problems, EMF-CE 
525 provided $0.16 \%, 0.80 \%, 3.09 \%, 4.62 \%$ and $4.51 \%$ improvements over LBSA, ASA-GS, HGA, GCGA and CONN to 526 the average shortest tour length, respectively. We can see that for the medium-scale TSPs, and the EMF-CE gave the 527 best tour length.

528

TABLE IV

529 COMPARING EMF-CE AND ITS FIVE COUNTERPARTS INCLUDING ASA-GS, GCGA, HGA, CONN AND 530 LBSA FOR THE SAME BENCHMARK TSPS FROM TSPLIB IN TERMS OF THE SOLUTION QUALITY AND 531 COMPUTATIONAL COMPLEXITY.

\begin{tabular}{|c|c|c|c|c|c|c|c|}
\hline \multirow{2}{*}{ Instances } & \multirow{2}{*}{ OPT } & EMF-CE & ASA-GS & GCGA & HGA & $\mathrm{CONN}$ & LBSA \\
\hline & & SL(\%) & $\mathrm{SL}(\%)$ & $\mathrm{SL}(\%)$ & $\mathrm{SL}(\%)$ & $\mathrm{SL}(\%)$ & $\mathrm{SL}(\%)$ \\
\hline eil51 & 426 & 0 & 0.67 & 0.23 & 0.67 & 2.58 & 0 \\
\hline st70 & 675 & 0 & 0.31 & 0 & 0.31 & 2.96 & 0 \\
\hline eil76 & 538 & 0 & 1.18 & 2.23 & 1.18 & 5.02 & 0 \\
\hline pr76 & 108159 & 0 & 0 & 0.14 & 0 & 4.34 & 0 \\
\hline rat99 & 1211 & 0 & 0.68 & 0.99 & 0.68 & 0.33 & 0 \\
\hline kroA100 & 21282 & 0 & 0.02 & 0.05 & 0.02 & 2.57 & 0 \\
\hline kroB100 & 22141 & 0 & 0 & 0.24 & na & 2.6 & 0 \\
\hline kroC100 & 20749 & 0 & 0.01 & 0.32 & 0.01 & 1.53 & 0 \\
\hline kroD100 & 21294 & 0 & 0 & 1.22 & 0 & 1.42 & 0 \\
\hline kroE100 & 22068 & 0 & 0.17 & 0.24 & na & 1.97 & 0 \\
\hline rd100 & 7910 & 0 & 0.01 & 0.96 & 0 & 3.59 & 0 \\
\hline eil101 & 629 & 0 & 1.78 & 1.59 & 1.78 & 5.09 & 0 \\
\hline $\operatorname{lin} 105$ & 14379 & 0 & 0.03 & 0.56 & 0.03 & 0.38 & 0 \\
\hline pr107 & 44303 & 0 & 0 & 1.15 & 0 & 2.77 & 0 \\
\hline pr124 & 59030 & 0 & 0 & 0 & 0 & 1.74 & 0 \\
\hline bier127 & 118282 & 0 & 0.01 & 0.91 & na & 2.45 & 0 \\
\hline $\operatorname{ch} 130$ & 6110 & 0 & 0.01 & na & 0.01 & 4.88 & 0 \\
\hline pr144 & 58537 & 0 & 0 & 0 & 0 & 2.34 & 0 \\
\hline $\operatorname{ch} 150$ & 6528 & 0 & 0.04 & na & 0.04 & 5.5 & 0 \\
\hline kroA150 & 26524 & 0 & 0 & 1.4 & 0 & 2.27 & 0 \\
\hline kroB150 & 26130 & 0 & 0.04 & 1.63 & - & 3.09 & 0 \\
\hline pr152 & 73682 & 0 & 0 & 0.19 & 0 & 0.79 & 0 \\
\hline u159 & 42080 & 0 & 0.74 & 1.74 & na & na & 0 \\
\hline kroA200 & 29368 & 0 & 0.15 & 1.33 & 0 & 0.79 & 0 \\
\hline kroB200 & 29437 & 0.01 & 0.23 & 2.09 & 0.05 & 1.07 & 0 \\
\hline ts 225 & 126643 & 0 & 0 & 0.64 & 1.18 & na & na \\
\hline pr226 & 80369 & 0 & 0.22 & 0.35 & 0.08 & 2.35 & na \\
\hline pr264 & 49135 & 0 & 0 & 0.62 & 0.03 & 3.58 & na \\
\hline a280 & 2579 & 0 & na & na & 3.13 & 4.24 & na \\
\hline pr299 & 48191 & 0.07 & 0.16 & 4.11 & 2.64 & 4.85 & 0 \\
\hline $\operatorname{lin} 318$ & 42029 & 0.3 & 0.66 & 3.74 & 1.42 & na & 0.1 \\
\hline $\mathrm{rd} 400$ & 15281 & 0.38 & 0.46 & 6.11 & 5.03 & 1.93 & 0.2 \\
\hline fl417 & 11861 & 0.1 & 0.67 & 1.65 & na & 3.58 & na \\
\hline
\end{tabular}




\begin{tabular}{cccccccc} 
pr439 & 107217 & 0.05 & 2.61 & 5.19 & 2.76 & 4.07 & na \\
pcb442 & 50778 & 0.46 & 0.56 & 7.66 & 4.13 & 4.8 & 0.11 \\
u574 & 36905 & 0.14 & 0.89 & na & na & 5.77 & na \\
u724 & 41910 & 0.12 & 0.87 & na & na & 6.48 & 0.7 \\
rat783 & 8806 & 0.45 & 1.68 & na & na & 6.72 & 0.92 \\
\multicolumn{2}{c}{ Average } & 0.05 & 0.4 & 1.54 & 0.87 & 3.16 & 0.07 \\
\hline
\end{tabular}

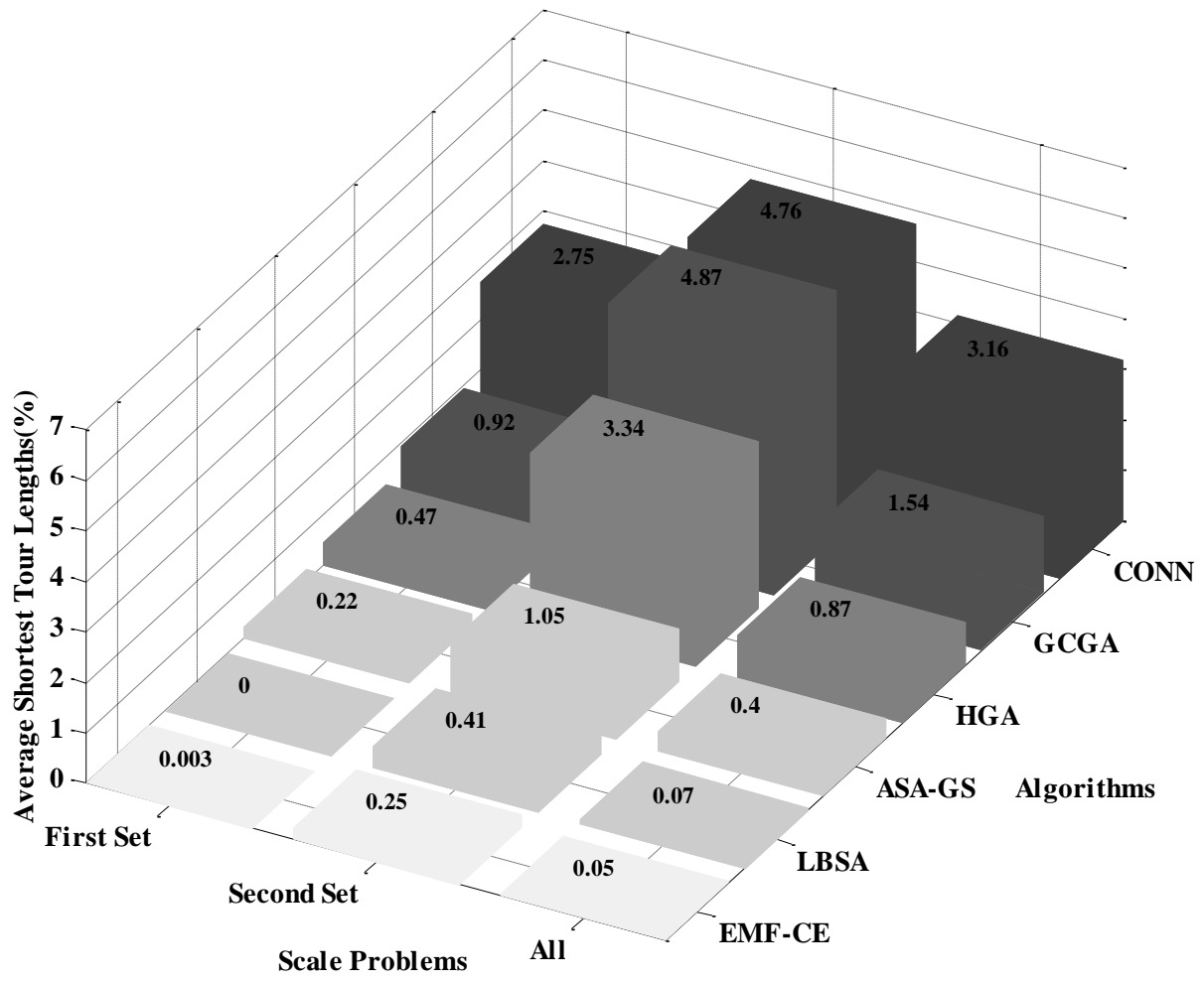

Fig.13 Average shortest tour lengths percent above optimality for EMF-CE and five comparable algorithms

\section{CONCLUSION}

In this paper, we presented a new method, which Contraction-expansion algorithm based on Move, Change, and Flip three operators called EMF-CE for TSPs. The main purpose of designing EMF-CE is to achieve near to optimal solution quality and the most accurate algorithm while the method would give comparatively slower of convergence speed. Its computational intensity is $O\left(n^{3}\right)$. But it is now can achieve global optimal for the small scale TSPs. For the generalized traveling salesman problems that round number and round integer may affect the end optimal result. 543 Usually can take 4 to 6 significant digit to calculate the distance and TSP path length, in order to prevent the loss of 544 precision and affect the realization of the optimal solution.

545 The EMF-CE performance was compared with those of several computationally comparable including ASA-GS, 546 GCGA, HGA, CONN, and LBSA, etc.. in terms of the qualitative comparison between those algorithms. However, 
547 we only show that five algorithms compares with the EMF-CE(have compared and reported in[25, 26, 38, 54], etc.). 548 Cause the others state-of-the-art (GATM, GSM, SA, ITS, GSA-ACS-PSO, RABNET-TSP, and MSA-IBS, etc. have 549 described and overviewed in section II) algorithm that can't give the best tour lengths (reach to global optimal) for 550 the small scale TSPs and let alone the medium-scale TSPs. Where EMF-CE has outperformed these state-of-the-art 551 NNs and evolution algorithms in terms of accuracy and in a reasonable time for the best tour length and average tour 552 length. But these algorithms provided a better and promising CPU time with poverty solution quality. Also, the CPU 553 time of EMF-CE with M code and only using single-core for computing. Moreover, the scaling coefficients large 554 than any 3G program language TABLE I. In other words, it is expected to obtain better performance by 555 implementing our algorithm using an efficient programming language like $\mathrm{C} / \mathrm{C}++$.

556 Future work should investigate and improve convergence speed. While the encouraging results presented here led 557 us to adapt the algorithm to solve more complex routing problems, such as both symmetric and asymmetric DTSPs, 558 and the capacitated vehicle routing problem (CVRP). Also, further investigations must be performed with larger TSP 559 instances and hybrid use of the proposed algorithm with route improvement heuristics, such as k-opt and simulated 560 annealing. 
582 [1] M. R. Garey and D. S. Johnson, Computers and Intractability: A Guide to the Theory of NP-Completeness. W. H. Freeman \\&amp; Co., 1979, p. 338.

[2] D. Gamboa, C. Rego, and F. Glover, "Implementation analysis of efficient heuristic algorithms for the traveling salesman problem," Computers \& Operations Research, vol. 33, no. 4, pp. 1154-1172, 4// 2006.

[3] D. Karapetyan and G. Gutin, "Lin-Kernighan heuristic adaptations for the generalized traveling salesman problem," European Journal of Operational Research, vol. 208, no. 3, pp. 221-232, 2/1/ 2011.

[4] W. Cook, "VLSI Data Sets," [Online], 2003.

[5] M. Fisher, "Chapter 1 Vehicle routing," in Handbooks in Operations Research and Management Science, vol. Volume 8: Elsevier, 1995, pp. 1-33.

[6] L. D. Whitley, T. Starkweather, and D. A. Fuquay, "Scheduling Problems and Traveling Salesmen: The Genetic Edge Recombination Operator," presented at the Proceedings of the 3rd International Conference on Genetic Algorithms, 1989.

[7] S. Kirkpatrick, C. D. Gelatt, and M. P. Vecchi, "Optimization by Simulated Annealing," Science, vol. 220, no. 4598, pp. 671-680, 1983.

[8] R. Agarwala, D. L. Applegate, D. Maglott, G. D. Schuler, and A. A. Schäffer, "A Fast and Scalable Radiation Hybrid Map Construction and Integration Strategy," Genome Research, vol. 10, no. 3, pp. 350-364, March 1, 20002000.

[9] P. Avner et al., "A radiation hybrid transcript map of the mouse genome," Nat Genet, 10.1038/ng1001-194 vol. 29, no. 2, pp. 194-200, 10//print 2001.

600

601

602

[10] F. Alizadeh, R. M. Karp, L. A. Newberg, and D. K. Weisser, "Physical mapping of chromosomes: A combinatorial problem in molecular biology," Algorithmica, vol. 13, no. 1, pp. 52-76, 1995// 1995.

[11] C. Korostensky and G. H. Gonnet, "Using traveling salesman problem algorithms for evolutionary tree construction," Bioinformatics, vol. 16, no. 7, pp. 619-627, July 1, 20002000.

[12] W. J. Cook, "In pursuit of the traveling salesman. Mathematics at the limits of computation," Princeton University Press, 2012.

606

607

[13] G. Tao and Z. Michalewicz, "Inver-over operator for the TSP," in Parallel Problem Solving from Nature PPSN V: 5th International Conference Amsterdam, The Netherlands September 27-30, 1998 Proceedings, A. E. Eiben, T. Bäck, M. Schoenauer, and H.-P. Schwefel, Eds. Berlin, Heidelberg: Springer Berlin Heidelberg, 1998, pp. 803-812.

[14] S. Lin and B. W. Kernighan, "An Effective Heuristic Algorithm for the Traveling-Salesman Problem," Operations Research, vol. 21, no. 2, pp. 498-516, 1973/04/01 1973.

[15] C. Rego, D. Gamboa, F. Glover, and C. Osterman, "Traveling salesman problem heuristics: Leading methods, implementations and latest advances," European Journal of Operational Research, vol. 211, no. 3, pp. 427441, 6/16/ 2011.

[16] M. Dorigo and L. M. Gambardella, "Ant colony system: a cooperative learning approach to the traveling salesman problem," IEEE Transactions on Evolutionary Computation, vol. 1, no. 1, pp. 53-66, 1997.

[17] N. L. J. Ulder, E. H. L. Aarts, H.-J. Bandelt, P. J. M. van Laarhoven, and E. Pesch, "Genetic local search algorithms for the traveling salesman problem," in Parallel Problem Solving from Nature: 1st Workshop, PPSN I Dortmund, FRG, October 1-3, 1990 Proceedings, H.-P. Schwefel and R. Männer, Eds. Berlin, Heidelberg: Springer Berlin Heidelberg, 1991, pp. 109-116.

[18] T. Huai-Kuang, Y. Jinn-Moon, T. Yuan-Fang, and K. Cheng-Yan, "An evolutionary algorithm for large traveling salesman problems," IEEE Transactions on Systems, Man, and Cybernetics, Part B (Cybernetics), vol. 34, no. 4, pp. 1718-1729, 2004.

[19] J. Hui-Dong, L. Kwong-Sak, W. Man-Leung, and Z. B. Xu, "An efficient self-organizing map designed by genetic algorithms for the traveling salesman problem," IEEE Transactions on Systems, Man, and Cybernetics, Part B (Cybernetics), vol. 33, no. 6, pp. 877-888, 2003.

[20] C. Contreras-Bolton and V. Parada, "Automatic Combination of Operators in a Genetic Algorithm to Solve the Traveling Salesman Problem," PLOS ONE, vol. 10, no. 9, p. e0137724, 2015. 
630 [21] J. Knox, "Heuristic, Genetic and Tabu SearchTabu search performance on the symmetric traveling salesman problem," Computers \& Operations Research, vol. 21, no. 8, pp. 867-876, 1994/10/01 1994.

[22] C. N. Fiechter, "A parallel tabu search algorithm for large traveling salesman problems," Discrete Applied Mathematics, vol. 51, no. 3, pp. 243-267, 1994/07/06 1994.

[23] E. Osaba, R. Carballedo, P. Lopez-Garcia, and F. Diaz, "Comparison between Golden Ball Meta-heuristic, Evolutionary Simulated Annealing and Tabu Search for the Traveling Salesman Problem," presented at the Proceedings of the 2016 on Genetic and Evolutionary Computation Conference Companion, Denver, Colorado, USA, 2016.

[24] J. M. Bostamam and Z. Othman, "Hybrid water flow-like algorithm with Tabu search for traveling salesman problem," AIP Conference Proceedings, vol. 1761, no. 1, p. 020058, 2016.

[25] X. Geng, Z. Chen, W. Yang, D. Shi, and K. Zhao, "Solving the traveling salesman problem based on an adaptive simulated annealing algorithm with greedy search," Applied Soft Computing, vol. 11, no. 4, pp. 3680-3689, 6// 2011.

[26] S.-M. Chen and C.-Y. Chien, "Solving the traveling salesman problem based on the genetic simulated annealing ant colony system with particle swarm optimization techniques," Expert Systems with Applications, vol. 38, no. 12, pp. 14439-14450, 11// 2011.

[27] Y. Wu, T. Weise, and R. Chiong, "Local search for the Traveling Salesman Problem: A comparative study," in Cognitive Informatics \& Cognitive Computing (ICCI $\left.{ }^{*} C C\right)$, 2015 IEEE 14th International Conference on, 2015, pp. 213-220.

[28] D. S. Johnson, The Traveling Salesman Problem: A Case Study in Local Optimization. 2010, pp. 215--310.

[29] M. Mavrovouniotis, M. F. M, X0OFc, ller, and S. Yang, "Ant Colony Optimization With Local Search for Dynamic Traveling Salesman Problems," IEEE Transactions on Cybernetics, vol. PP, no. 99, pp. 1-14, 2016.

[30] M. Al-Mulhem and T. Al-Maghrabi, "Efficient convex-elastic net algorithm to solve the Euclidean traveling salesman problem," IEEE Transactions on Systems, Man, and Cybernetics, Part B (Cybernetics), vol. 28, no. 4, pp. 618-620, 1998.

[31] B. Avşar and D. E. Aliabadi, "Parallelized neural network system for solving Euclidean traveling salesman problem," Applied Soft Computing, vol. 34, pp. 862-873, 9// 2015.

[32] L. Buriol, P. M. França, and P. Moscato, "A New Memetic Algorithm for the Asymmetric Traveling Salesman Problem," Journal of Heuristics, vol. 10, no. 5, pp. 483-506, 2004// 2004.

[33] B. Freisleben and P. Merz, "A genetic local search algorithm for solving symmetric and asymmetric traveling salesman problems," in Evolutionary Computation, 1996., Proceedings of IEEE International Conference on, 1996, pp. 616-621.

[34] X. Yang and J. s. Wang, "Application of improved ant colony optimization algorithm on traveling salesman problem," in 2016 Chinese Control and Decision Conference (CCDC), 2016, pp. 2156-2160.

[35] T. Saenphon, S. Phimoltares, and C. Lursinsap, "Combining new Fast Opposite Gradient Search with Ant Colony Optimization for solving travelling salesman problem," Engineering Applications of Artificial Intelligence, vol. 35, pp. 324-334, 10// 2014.

[36] T. A. S. Masutti and L. N. de Castro, "A self-organizing neural network using ideas from the immune system to solve the traveling salesman problem," Information Sciences, vol. 179, no. 10, pp. 1454-1468, 4/29/ 2009.

[37] Y.-F. Liao, D.-H. Yau, and C.-L. Chen, "Evolutionary algorithm to traveling salesman problems," Computers \& Mathematics with Applications, vol. 64, no. 5, pp. 788-797, 9// 2012.

[38] M. Mahi, Ö. K. Baykan, and H. Kodaz, "A new hybrid method based on Particle Swarm Optimization, Ant Colony Optimization and 3-Opt algorithms for Traveling Salesman Problem," Applied Soft Computing, vol. 30, pp. 484-490, 5// 2015.

[39] G. Vahdati, M. Yaghoubi, M. Poostchi, and S. M. B. N, "A New Approach to Solve Traveling Salesman Problem Using Genetic Algorithm Based on Heuristic Crossover and Mutation Operator," in Soft Computing and Pattern Recognition, 2009. SOCPAR '09. International Conference of, 2009, pp. 112-116.

[40] K. S. Leung, H. D. Jin, and Z. B. Xu, "An expanding self-organizing neural network for the traveling salesman problem," Neurocomputing, vol. 62, no. 1, pp. 267-292, 2004. 
[41] M. Budinich, "A Self-Organizing Neural Network for the Traveling Salesman Problem That Is Competitive with Simulated Annealing," Neural Computation, vol. 8, no. 2, pp. 416-424, 1996/02/15 1996.

[42] B. Fritzke and P. Wilke, "FLEXMAP-a neural network for the traveling salesman problem with linear time and space complexity," in Neural Networks, 1991. 1991 IEEE International Joint Conference on, 1991, pp. 929-934 vol.2.

[43] H.-M. Feng and K.-L. Liao, "Hybrid evolutionary fuzzy learning scheme in the applications of traveling salesman problems," Information Sciences, vol. 270, pp. 204-225, 6/20/ 2014.

[44] R. Baraglia, J. I. Hidalgo, and R. Perego, "A hybrid heuristic for the traveling salesman problem," IEEE Transactions on Evolutionary Computation, vol. 5, no. 6, pp. 613-622, 2001.

[45] H. D. Nguyen, I. Yoshihara, K. Yamamori, and M. Yasunaga, "Implementation of an Effective Hybrid GA for Large-Scale Traveling Salesman Problems," IEEE Transactions on Systems, Man, and Cybernetics, Part B (Cybernetics), vol. 37, no. 1, pp. 92-99, 2007.

[46] J. W. Pepper, B. L. Golden, and E. A. Wasil, "Solving the traveling salesman problem with annealing-based heuristics: a computational study," IEEE Transactions on Systems, Man, and Cybernetics - Part A: Systems and Humans, vol. 32, no. 1, pp. 72-77, 2002.

[47] Y. Li, V. A. Protopopescu, N. Arnold, X. Zhang, and A. Gorin, "Hybrid parallel tempering and simulated annealing method," Applied Mathematics and Computation, vol. 212, no. 1, pp. 216-228, 6/1/ 2009.

[48] C. Y. Wang, M. Lin, Y. W. Zhong, and H. Zhang, "Solving travelling salesman problem using multiagent simulated annealing algorithm with instance-based sampling," International Journal of Computing Science \& Mathematics, vol. 6, no. 4, pp. 336-353, 2015.

[49] Y. Zhong, L. Wang, C. Wang, and H. Zhang, "Multi-agent simulated annealing algorithm based on differential evolution algorithm," International Journal of Bio-Inspired Computation, vol. 4, no. 4, pp. 217-228, 2012.

[50] M. Zlochin, M. Birattari, N. Meuleau, and M. Dorigo, "Model-Based Search for Combinatorial Optimization: A Critical Survey," Annals of Operations Research, vol. 131, no. 1, pp. 373--395, 2004.

[51] C. Wang, M. Lin, Y. Zhong, and H. Zhang, "Swarm simulated annealing algorithm with knowledge-based sampling for travelling salesman problem," International Journal of Intelligent Systems Technologies \& Applications, vol. 15, no. 1, pp. 74-94, 2016.

[52] C. D. Tarantilis and C. T. Kiranoudis, "A list-based threshold accepting method for job shop scheduling problems," International Journal of Production Economics, vol. 77, no. 2, pp. 159-171, 2002.

[53] C. D. Tarantilis, C. T. Kiranoudis, and V. S. Vassiliadis, "A list based threshold accepting metaheuristic for the heterogeneous fixed fleet vehicle routing problem," Journal of the Operational Research Society, vol. 54, no. 54, pp. 65-71(7), 2003.

[54] S. H. Zhan, J. Lin, Z. J. Zhang, and Y. W. Zhong, "List-Based Simulated Annealing Algorithm for Traveling Salesman Problem," Computational Intelligence \& Neuroscience, vol. 2016, no. 5, pp. 1-12, 2016.

[55] A. Colorni, M. Dorigo, and V. Maniezzo, "Distributed Optimization by Ant Colonies," in European Conference on Artificial Life, 1991.

[56] S. C. Chu, J. F. Roddick, and J. S. Pan, "Ant colony system with communication strategies," (in English), Information Sciences, Article vol. 167, no. 1-4, pp. 63-76, Dec 2004.

[57] S.-M. Chen and C.-Y. Chien, "Parallelized genetic ant colony systems for solving the traveling salesman problem," Expert Systems with Applications, vol. 38, no. 4, pp. 3873-3883, 4// 2011.

[58] M. Mavrovouniotis and S. Yang, "Ant colony optimization with direct communication for the traveling salesman problem," in 2010 UK Workshop on Computational Intelligence (UKCI), 2010, pp. 1-6.

[59] T. Stützle and H. H. Hoos, "The MAX-MIN ant system and local search for the traveling salesman problem," 1997.

[60] K. Helsgaun, "An effective implementation of the Lin-Kernighan traveling salesman heuristic," European Journal of Operational Research, vol. 126, no. 1, pp. 106-130, 2000.

[61] K. Helsgaun, "General k-opt submoves for the Lin-Kernighan TSP heuristic," Mathematical Programming Computation, vol. 1, no. 2, pp. 119-163, 2009// 2009.

[62] M. Saadatmand-Tarzjan, M. Khademi, M. R. Akbarzadeh-T, and H. A. Moghaddam, "A Novel ConstructiveOptimizer Neural Network for the Traveling Salesman Problem," IEEE Transactions on Systems, Man, and Cybernetics, Part B (Cybernetics), vol. 37, no. 4, pp. 754-770, 2007. 
[63] Y. Takahashi, "Mathematical improvement of the Hopfield model for feasible solutions to the traveling salesman problem by a synapse dynamical system," IEEE Transactions on Cybernetics, vol. 28, no. 6, pp. 906-19, 1998.

[64] R. Pasti and L. N. De Castro, "A Neuro-Immune Network for Solving the Traveling Salesman Problem," in International Joint Conference on Neural Networks, 2006, pp. 3760-3766.

[65] GU Shi-Liang, WAN Lin-Sheng, HUANG Li-Juan, and WANG Wen-Ping, "Improved Contraction-Expansion Algorithm for Curve and Surface Fitting," Acta Agronomica Sinica, no. 04, pp. 583-589, 2007.

[66] K. Helsgaun, "An Effective Implementation of K-opt Moves for the Lin-Kernighan TSP Heuristic," Transactions of the Japan Society of Mechanical Engineers A, vol. 69, no. 12, pp. 1615-1628, 2010.

[67] G. Reinelt, "TSPLIB-A Traveling Salesman Problem Library," ORSA Journal on Computing, vol. 3, no. 4, pp. 376-384, 1991.

[68] J. Yang, C. Wu, H. P. Lee, and Y. Liang, "Solving traveling salesman problems using generalized chromosome genetic algorithm," Progress in Natural Science, vol. 18, no. 7, pp. 887-892, 7/10/ 2008.

[69] Y. Wang, "The hybrid genetic algorithm with two local optimization strategies for traveling salesman problem," Computers \& Industrial Engineering, vol. 70, pp. 124-133, 4// 2014.

[70] M. Albayrak and N. Allahverdi, "Development a new mutation operator to solve the Traveling Salesman Problem by aid of Genetic Algorithms," Expert Systems with Applications, vol. 38, no. 3, pp. 1313-1320, 3// 2011.

[71] D. S. Johnson and L. A. McGeoch, "Experimental Analysis of Heuristics for the STSP," in The Traveling Salesman Problem and Its Variations, G. Gutin and A. P. Punnen, Eds. Boston, MA: Springer US, 2007, pp. 369-443. 